\title{
Rees algebras on smooth schemes: integral closure and higher differential operator
}

\section{Orlando Villamayor U.}

\begin{abstract}
Let $V$ be a smooth scheme over a field $k$, and let $\left\{I_{n}, n \geq 0\right\}$ be a filtration of sheaves of ideals in $\mathcal{O}_{V}$, such that $I_{0}=\mathcal{O}_{V}$, and $I_{s} \cdot I_{t} \subset I_{s+t}$. In such case $\bigoplus I_{n}$ is called a Rees algebra.

A Rees algebra is said to be a differential algebra if, for any two integers $N>n$ and any differential operator $D$ of order $n, D\left(I_{N}\right) \subset$ $I_{N-n}$. Any Rees algebra extends to a smallest differential algebra.

There are two extensions of Rees algebras of interest in singularity theory: one defined by taking integral closures, and another by extending the algebra to a differential algebra.

We study here some relations between these two extensions, with particular emphasis on the behavior of higher order differentials over arbitrary fields.
\end{abstract}

\section{Introduction}

A smooth ring $R$, of finite type over a field $k$, has a locally free sheaf of $k$-linear differential of order $n$, say $\operatorname{Diff}^{n}(R)$, for each index $n \geq 0$.

A graded subring of a polynomial ring over $R$, say $R[W]$, can be expressed as $\mathcal{G}=\bigoplus_{n>0} I_{n} \cdot W^{n}$, where each $I_{n}$ is an ideal in $R$. $\mathcal{G}$ is called the Rees algebra of the filtration $\left\{I_{n}\right\}_{n>0}$. For example a Rees ring of an ideal $I$ is of this type; in which case $I_{n}=I^{n}$. The integral closure of a Rees ring is also a Rees algebra (not necessarily a Rees ring of an ideal).

Taking integral closure of Rees algebras in $R[W]$ can be thought of as an operator, say $\mathcal{G} \subset \overline{\mathcal{G}}$.

2000 Mathematics Subject Classification: 14E15.

Keywords: Integral closure, Rees algebras. 
The study of embedded singularities has motivated another kind of extension, linked to differential operators. In fact, from the point of view of singularities it is interesting to consider Rees algebras $\mathcal{G}=\bigoplus_{n \geq 0} I_{n} \cdot W^{n}$ with the additional compatibility with differential operators; namely with the property that for any operator $D \in \operatorname{Diff}^{n}(R)$, and any index $N>n$, $D\left(I_{N}\right) \subset I_{N-n}$.

It is simple to show that for any $\mathcal{G}=\bigoplus_{n>0} I_{n} \cdot W^{n}$, there is a smallest extension to one with this property, say $G(\mathcal{G})$. This defines a second operator, say $\mathcal{G} \subset G(\mathcal{G})$. The objective of this paper is to study the interplay between both operators. In Theorem 6.13 it is shown that if two Rees algebras have the same integral closure, then their $G$-extensions also have the same integral closure. This shows a curious relation of differential operators with integral closures.

Sections 2 and 3 are devoted to extensions of Rees algebras to those which are compatible with differential operators.

In Sections 4 and 5 we study some natural relation of of the first two sections with the study of singularities.

Our main results are addressed in Section 6. The key idea is to consider suitable weighted algebras defined by coefficients of truncated Taylor development. In fact, it is in this context where the link of integral closure with differential operators arises.

The study of differential operators on smooth schemes, and their relation with with integral closure have also been developed independently in a very interesting paper of Kawanoue, including Theorem 6.13 (see [9]).

The results in this paper have been applied in the study of singularities over arbitrary fields. In fact, these results were essential in order to develop a notion of elimination, defined in terms of Rees algebras; a notion with application to the study of singularities of hypersurfaces over fields of positive characteristic (see [19]).

Extensions of algebras by differential operators appeared in [22], and more recently in work of J. Wlodarczyk and J. Kollár ([10] and [20]). But it is in the work of Hironaka $[6,7,8]$ where the notion of differential algebra is treated systematically in relation to the theory of infinitely close points. Our work is related with these last three papers, particularly with his "finite presentation theorem" in [7]. In this paper we do not make use of monoidal transformations, and hence of the theory of infinitely close points. The notion of restriction of differential algebras, in section 5, appears already in the work of Hironaka. We refer here to [1] and [18] for other applications of this work, and also for more details on the relation of our results with results of Hironaka.

I profited from discussions with Vincent Cossart, Marco Farinati, Monique Lejeune, Luis Narváez, Augusto Nobile, and Santiago Zarzuela. 


\section{Graded rings and Differential algebras}

2.1. Fix a noetherian $\operatorname{ring} B$, and a sequence of ideals $\left\{I_{k}\right\}, k \geq 0$, which fulfill the conditions:

1) $I_{0}=B$, and

2) $I_{k} \cdot I_{s} \subset I_{k+s}$.

This defines a graded subring $\mathcal{G}=\bigoplus_{k \geq 0} I_{k} \cdot W^{k}$ of the polynomial ring $B[W]$. We say that $\mathcal{G}$ is a Rees algebra if this subring is a (noetherian) finitely generated $B$-algebra.

Remark 2.2. 1) Examples of Rees algebras are Rees rings of ideals, say $I \subset B$, where $I_{k}=I^{k}$ for each $k \geq 1$. In general we will not assume that a Rees algebra is generated in degree one.

2) Whenever $\bigoplus I_{k} \cdot W^{k}(\subset B[W])$ is a Rees algebra, a new Rees algebra $\bigoplus I_{k}^{\prime} \cdot W^{k}$ is defined by setting

$$
I_{k}^{\prime}=\sum_{r \geq k} I_{r}
$$

If $\bigoplus I_{k} \cdot W^{k}$ is generated by $\mathcal{F}=\left\{g_{n_{i}} \cdot W^{n_{i}} \mid 1 \leq i \leq m, n_{i}>0\right\}$. Namely, if:

$$
\bigoplus I_{k} \cdot W^{k}=B\left[\left\{g_{n_{i}} \cdot W^{n_{i}}\right\}_{g_{n_{i}} \cdot W^{n_{i} \in \mathcal{F}}}\right]
$$

then $\bigoplus I_{k}^{\prime} \cdot W^{k}$ is generated by the finite set $\left\{g_{n_{i}} \cdot W^{n_{i}^{\prime}} \mid 1 \leq i \leq m, 1 \leq n_{i}^{\prime} \leq n_{i}\right\}$.

Note that $I_{k}^{\prime} \supset I_{k+1}^{\prime}$, and that $\bigoplus I_{k} \cdot W^{k} \subset \bigoplus I_{k}^{\prime} \cdot W^{k}$ is a finite extension. In fact, it suffices to check that given an element $g \in I_{k}$, then $g \cdot W^{k-1}$ is integral over $\bigoplus I_{k} \cdot W^{k}$. One can check that

$$
g \in I_{k} \Rightarrow g^{k-1} \in I_{k(k-1)} \Rightarrow g^{k} \in I_{k(k-1)},
$$

so $g \cdot W^{k-1}$ fulfills the equation $Z^{k}-\left(g^{k} \cdot W^{k(k-1)}\right)=0$.

In this paper we shall consider Rees algebras up to integral closure, so in the sequel we will assume that:

$$
I_{k} \supset I_{k+1} .
$$

2.3. In what follows we define a Rees algebra, say $\bigoplus_{n \geq 0} I_{n} \cdot W^{n}$ in $B[W]$, by fixing a set of generators, say

$$
\mathcal{F}=\left\{g_{n_{i}} \cdot W^{n_{i}} \mid n_{i}>0,1 \leq i \leq m\right\}
$$

So if $f \in I_{n}$, then

$$
f=F_{n}\left(g_{n_{1}}, \ldots, g_{n_{m}}\right),
$$

where $F_{n}\left(Y_{1}, \ldots, Y_{m}\right)$ is a weighted homogeneous polynomial in $m$ variables, and each $Y_{j}$ has weight $n_{j}$. 
For a fixed positive integer $N, B\left[W^{N}\right] \subset B[W]$ is a finite extension of graded rings. Furthermore, $\bigoplus_{k>0} I_{k N} \cdot W^{k N}$ is a Rees algebra in $B\left[W^{N}\right]$, and the ring extension $\bigoplus_{k \geq 0} I_{k N} \cdot W^{k N} \subset \bigoplus_{n \geq 0} I_{n} \cdot W^{n}$ is also finite.

Note that if $N$ is a common multiple of all integers $n_{i}, 1 \leq i \leq m$, then

$$
\bigoplus_{k \geq 0} I_{N}^{k} \cdot W^{k N} \subset \bigoplus_{n \geq 0} I_{n} \cdot W^{n}
$$

is an integral extension, where the left hand side is the Rees ring of $I_{N}$ (in $\left.B\left[W^{N}\right]\right)$. So Rees algebras are finite extensions of Rees rings.

If a Rees algebra $\bigoplus_{n \geq 0} I_{n} \cdot W^{n}$ in $B[W]$ is the Rees ring of $I_{1}$, then the integral closure in $B[W]$ is $\bigoplus_{n>0} \bar{I}_{n} \cdot W^{n}$, where each $\bar{I}_{n}$ is the integral closure of the ideal $I_{n}$. This is a Rees algebra, and not necessarily the Rees ring of the ideal $\bar{I}_{1}$.

2.4. Let $B$ be a normal excellent ring, and let

$$
\operatorname{Spec}(B) \stackrel{\pi}{\longleftarrow} X
$$

be a proper birational morphism, then $I \subset \pi_{*}\left(I \mathcal{O}_{X}\right) \subset \bar{I}$, where $\bar{I}$ denotes the integral closure of $I$ in $B$. Moreover, if $\pi$ is the normalization of the blow-up at $I$, then $I \mathcal{O}_{X}$ is an invertible sheaf of ideals, and

$$
\bar{I}=\pi_{*}\left(I \mathcal{O}_{X}\right) .
$$

Assume that the normal $\operatorname{ring} B$ is of finite type over a field $k$. If $B$ is a one dimensional normal domain, any ideal is invertible and integrally closed. We add the following well known result for self-containment (see [6, p. 54] or $[12$, p. 100]).

Lemma 2.5. Let $I, J$ be two ideals in a normal domain $B$, which is finitely generated over a field $k$. Then $\bar{I}=\bar{J}$ if and only if $I \mathcal{O}_{W}=J \mathcal{O}_{W}$, for any morphism of $k$-schemes $W \rightarrow \operatorname{Spec}(B)$, with $W$ of dimension one, regular and of finite type over $k$.

Proof. Let $x \in W$ be a closed point that maps to $y \in \operatorname{Spec}(B)$, then $\mathcal{O}_{W, x}$ is a valuation ring that dominates $\mathcal{O}_{\operatorname{Spec}(B), y}$. So if $\bar{I}=\bar{J}$, then $I \mathcal{O}_{W}=J \mathcal{O}_{W}$. In fact, for any morphism $B \rightarrow A$, where $A$ is a valuation ring, $I A=\bar{I} A$.

Assume that this condition holds for any morphism from a regular one dimensional scheme $W$. We claim now that both ideals have the same integral closure in $B$.

Let $\operatorname{Spec}(B) \stackrel{\pi}{\longleftarrow} X$ be the normalized blow up at $I$, and let $\left\{H_{1}, \ldots, H_{s}\right\}$ be the irreducible components of the closed set defined by the invertible sheaf of ideals $I \mathcal{O}_{X}$. Here each $H_{i}$ is an irreducible hypersurfaces in $X$. Let $h_{i} \in X$ denote the generic point of $H_{i}$. There are positive integers $a_{i}$, so that $I \mathcal{O}_{X}$ 
can be characterized as the sheaf of functions vanishing along $H_{i}$ with order at least $a_{i}$ (i.e. with order at least $a_{i}$ at the valuation rings $\mathcal{O}_{X, h_{i}}$ ).

Claim: The sheaf of ideals $J \mathcal{O}_{X}$ also has order $a_{i}$ at $\mathcal{O}_{X, h_{i}}$.

If the claim holds, $J \mathcal{O}_{X} \subset I \mathcal{O}_{X}$, and

$$
J \subset \pi_{*}\left(J \mathcal{O}_{X}\right) \subset \pi_{*}\left(I \mathcal{O}_{X}\right)=\bar{I} .
$$

In particular $\bar{J} \subset \bar{I}$. A similar argument would lead to the other inclusion.

In order to prove the claim we choose a closed point $x \in H_{i}$ so that:

1) $\mathcal{O}_{X, x}$ is regular,

2) $x \in H_{i}-\cap_{j \neq i} H_{j}$,

3) $H_{i}$ is regular at $x$, and

4) $J \mathcal{O}_{X, x}$ is a $p$-primary ideal, for $p=I\left(H_{i}\right)_{x}$.

Since any sheaf of ideals has only finitely many $p$-primary components, such choice of $x$ is possible.

Let $\left\{x_{1}, \ldots x_{d-1}, x_{d}\right\}$ be a regular system of parameters at $\mathcal{O}_{X, x}$ such that $p=I\left(H_{i}\right)_{x}=x_{d} \mathcal{O}_{X, x}$, and let $W$ be the closure of the irreducible curve defined locally by $\left\langle x_{1}, \ldots, x_{d-1}\right\rangle$. So $W$ is one dimensional, and regular locally at $x$. We may assume that $W$ is regular after applying quadratic transformations which do not affect the local ring $\mathcal{O}_{W, x}$. By construction $I \mathcal{O}_{W, x}$ has order $a_{i}$, by hypothesis the same holds for $J \mathcal{O}_{W, x}$. This proves the claim.

2.6. Let $B=S[X]$ be a polynomial ring, and let Tay : $B \rightarrow B[U]$ be the $S$-algebra homomorphism defined by setting Tay $(X)=X+U$. For any $f(X) \in B$ set

$$
\operatorname{Tay}(f(X))=\sum_{\alpha \geq 0} \Delta^{\alpha}(f(X)) U^{\alpha} .
$$

The operators $\Delta^{\alpha}$ are $S$-differential operators ( $S$ linear). Furthermore, for any positive integer $N$, the set $\left\{\Delta^{\alpha} \mid 0 \leq \alpha \leq N\right\}$ is a basis of the $B$ module of $S$-differential operators on $B$, of order $\leq N$.

Definition 2.7. Let $B=S[X]$ be a polynomial ring over a noetherian ring $S$. A Rees algebra

$$
\bigoplus I_{k} \cdot W^{k} \subset B[W]
$$

is a differential algebra, say Diff-algebra, relative to $S$, when:

i) $I_{k} \supset I_{k+1}$ for any $k \geq 0$.

ii) For any $n>0$ and $f \in I_{n}$, and for any index $0 \leq j \leq n$ and any $S$-differential operator of order $\leq j$, say $D_{j}$ :

$$
D_{j}(f) \in I_{n-j} .
$$


Remark 2.8. Let $\operatorname{Diff}_{S}^{N}(B)$ denote the module of $S$-differential operators of order at most $N$. Then

$$
\operatorname{Diff}_{S}^{N}(B) \subset \operatorname{Diff}_{S}^{N+1}(B) \subset \ldots
$$

For this reason it is natural to require condition (i) in our previous definition. Note also that 2.6 asserts that (ii) can be reformulated as:

ii') For any $n>0$ and $f \in I_{n}$, and for any index $0 \leq \alpha \leq n$ :

$$
\Delta^{\alpha}(f) \in I_{n-\alpha}
$$

In fact, (i) and (ii) are equivalent to (i) and (ii'):

Theorem 2.9. Fix $B=S[X]$ as before, and a finite set $\mathcal{F}=\left\{g_{n_{i}} W^{n_{i}} \mid n_{i}>\right.$ $0,1 \leq i \leq m\}$, with the following properties:

a) For any $1 \leq i \leq m$, and any $n_{i}^{\prime}, 0<n_{i}^{\prime} \leq n_{i}$ :

$$
g_{n_{i}} \cdot W^{n_{i}^{\prime}} \in \mathcal{F} \text {. }
$$

b) For any $1 \leq i \leq m$, and for any index $0 \leq \alpha<n_{i}$ :

$$
\Delta^{\alpha}\left(g_{n_{i}}\right) \cdot W^{n_{i}-\alpha} \in \mathcal{F} .
$$

Then the $B$ sub-algebra of $B[W]$, generated by $\mathcal{F}$ over the ring $B$, is a Diff-algebra relative to $S$.

Proof. Condition (i) in Definition 2.7 holds by 2.2, 2).

Fix a positive integer $N$, and let $I_{N} \cdot W^{N}$ be the homogeneous component of degree $N$ of the $B$ sub-algebra generated by $\mathcal{F}$. We prove that for any $h \in I_{N}$, and any $0 \leq \alpha \leq N, \Delta^{\alpha}(h) \in I_{N-\alpha}$.

The ideal $I_{N} \subset B$ is generated by all elements of the form

$$
H_{N}=g_{n_{i_{1}}} \cdot g_{n_{i_{2}}} \cdots g_{n_{i_{p}}} \quad n_{i_{1}}+n_{i_{2}}+\cdots n_{i_{p}}=N
$$

with the $g_{n_{i_{j}}} \cdot W^{n_{i_{j}}} \in \mathcal{F}$ not necessarily different.

Since the operators $\Delta^{\alpha}$ are linear, it suffices to prove that $\Delta^{\alpha}\left(a \cdot H_{N}\right) \in$ $I_{N-\alpha}$, for $a \in B, H_{N}$ as in 2.9.1, and $0 \leq \alpha \leq N$. We proceed in two steps, by proving:

1) $\Delta^{\alpha}\left(H_{N}\right) \in I_{N-\alpha}$.

2) $\Delta^{\alpha}\left(a \cdot H_{N}\right) \in I_{N-\alpha}$.

We first prove 1). Set Tay : $B=S[X] \rightarrow B[U]$, as in 2.6. Consider, for any element $g_{n_{i_{l}}} \cdot W^{n_{i_{l}}} \in \mathcal{F}$,

$$
\operatorname{Tay}\left(g_{n_{i_{l}}}\right)=\sum_{\beta \geq 0} \Delta^{\beta}\left(g_{n_{i_{l}}}\right) U^{\beta} \in B[U]
$$


Hypothesis (b) states that for each $0 \leq \beta<n_{i_{l}}, \Delta^{\beta}\left(g_{n_{i_{l}}}\right) \cdot W^{n_{i_{l}}-\beta} \in \mathcal{F}$. On the one hand

$$
\operatorname{Tay}\left(H_{N}\right)=\sum_{\alpha \geq 0} \Delta^{\alpha}\left(H_{N}\right) U^{\alpha}
$$

and, on the other hand

$$
\operatorname{Tay}\left(H_{N}\right)=\operatorname{Tay}\left(g_{n_{i_{1}}}\right) \cdot \operatorname{Tay}\left(g_{n_{i_{2}}}\right) \cdots \operatorname{Tay}\left(g_{n_{i_{p}}}\right)
$$

in $B[U]$. This shows that for a fixed $\alpha(0 \leq \alpha \leq N), \Delta^{\alpha}\left(H_{N}\right)$ is a sum of elements of the form:

$$
\Delta^{\beta_{1}}\left(g_{n_{i_{1}}}\right) \cdot \Delta^{\beta_{2}}\left(g_{n_{i_{2}}}\right) \cdots \Delta^{\beta_{p}}\left(g_{n_{i_{p}}}\right), \quad \sum_{1 \leq s \leq p} \beta_{s}=\alpha .
$$

So it suffices to show that each of these summands is in $I_{N-\alpha}$.

Note here that

$$
\sum_{1 \leq s \leq p}\left(n_{i_{s}}-\beta_{s}\right)=N-\alpha
$$

and that some of the integers $n_{i_{s}}-\beta_{s}$ might be zero or negative. Set

$$
G=\left\{r \mid 1 \leq r \leq p \text { and } n_{i_{r}}-\beta_{r}>0\right\} .
$$

So

$$
N-\alpha=\sum_{1 \leq s \leq p}\left(n_{i_{s}}-\beta_{s}\right) \leq \sum_{r \in G}\left(n_{i_{r}}-\beta_{r}\right)=M .
$$

Hypothesis (b) ensures that $\Delta^{\beta_{r}}\left(g_{n_{i_{r}}}\right) \in I_{n_{i_{r}}-\beta_{r}}$ for every index $r \in G$, in particular:

$$
\Delta^{\beta_{1}}\left(g_{n_{i_{1}}}\right) \cdot \Delta^{\beta_{2}}\left(g_{n_{i_{2}}}\right) \cdots \Delta^{\beta_{p}}\left(g_{n_{i_{p}}}\right) \in I_{M} .
$$

Finally, since $M \geq N-\alpha, I_{M} \subset I_{N-\alpha}$, and this proves Case 1$)$.

For Case 2), fix $0 \leq \alpha \leq N$. We claim that $\Delta^{\alpha}\left(a \cdot H_{N}\right) \in I_{N-\alpha}$, for $a \in B$ and $H_{N}$ as in 2.9.1. At the ring $B[U]$,

$$
\operatorname{Tay}\left(a \cdot H_{N}\right)=\sum_{\alpha \geq 0} \Delta^{\alpha}\left(a \cdot H_{N}\right) U^{\alpha},
$$

and, on the other hand

$$
\operatorname{Tay}\left(a \cdot H_{N}\right)=\operatorname{Tay}(a) \cdot \operatorname{Tay}\left(H_{N}\right) .
$$

This shows that $\Delta^{\alpha}\left(a \cdot H_{N}\right)$ is a sum of terms of the form $\Delta^{\alpha_{1}}(a) \cdot \Delta^{\alpha_{2}}\left(H_{N}\right)$, $\alpha_{i} \geq 0$, and $\alpha_{1}+\alpha_{2}=\alpha$. In particular $\alpha_{2} \leq \alpha$; and by Case 1$), \Delta^{\alpha_{2}}\left(H_{N}\right) \in$ $I_{N-\alpha_{2}}$. On the other hand $N-\alpha_{2} \geq N-\alpha$, so $\Delta^{\alpha_{2}}\left(H_{N}\right) \in I_{N-\alpha}$, and hence $\Delta^{\alpha}\left(a \cdot H_{N}\right) \in I_{N-\alpha}$. 
Corollary 2.10. The Rees algebra in $B[W]$, generated over $B$ by

$$
\mathcal{F}=\left\{g_{n_{i}} \cdot W^{n_{i}} \mid n_{i}>0,1 \leq i \leq m\right\}
$$

extends to a smallest Diff-algebra, which is generated by the finite set

$$
\mathcal{F}^{\prime}=\left\{\Delta^{\alpha}\left(g_{n_{i}}\right) \cdot W^{n_{i}^{\prime}-\alpha} \mid g_{n_{i}} \cdot W^{n_{i}} \in \mathcal{F} \text {, and } 0 \leq \alpha<n_{i}^{\prime} \leq n_{i}\right\}
$$

Remark 2.11. Theorem 2.9 shows how to extend any Rees algebra to a Diff-algebra, say $\bigoplus I_{k} \cdot W^{k} \subset B[W]$ so that the conditions of Definition 2.7 holds; namely that for any $S$-differential operator of order $j(\leq n)$, say $D_{j}$ : $D_{j}\left(I_{n}\right) \in I_{n-j}$.

A similar argument can be used to extend Rees algebras to algebras, say $\bigoplus I_{k} \cdot W^{k} \subset B[W]$ again, with the condition:

$$
D_{j}\left(I_{n}\right) \in I_{n}
$$

for any positive $n$, and any differential operator of order $j$, with no condition on $j$. It is easy to check that ideals $I_{n}$ with this property are those generated by elements in $S$.

Consider, as in Theorem 2.9, a finite set $\mathcal{F}=\left\{g_{n_{i}} \cdot W^{n_{i}}, n_{i}>0,1 \leq i \leq m\right\}$ with the following properties:

a) For any $1 \leq i \leq m$, and any $n_{i}^{\prime}, 0<n_{i}^{\prime} \leq n_{i}: g_{n_{i}} W^{n_{i}^{\prime}} \in \mathcal{F}$.

b) For any $1 \leq i \leq m$, and for any index $0 \leq \alpha$ : $\Delta^{\alpha}\left(g_{n_{i}}\right) W^{n_{i}} \in \mathcal{F}$.

We claim now that the $B$ sub-algebra of $B[W]$, generated by $\mathcal{F}$ over the ring $B$, fulfills (2.11.1). Note here that as each $g_{n_{i}}$ is polynomial on $Z$, $\Delta^{\alpha}\left(g_{n}\right)=0$ for $\alpha$ big enough, so $\mathcal{F}$ is in fact finite.

In order to prove the claim it suffices to show that $\Delta^{\alpha}\left(a \cdot H_{N}\right) \in I_{N}$, for $a \in B, H_{N}$ as in (2.9.1). As in the previous Theorem we proceed in two steps, but proving now that:

1) $\Delta^{\alpha}\left(H_{N}\right) \in I_{N}$.

2) $\Delta^{\alpha}\left(a \cdot H_{N}\right) \in I_{N}$.

$\Delta^{\alpha}\left(H_{N}\right)$ is a sum of elements of the form:

$$
\Delta^{\beta_{1}}\left(g_{n_{i_{1}}}\right) \cdot \Delta^{\beta_{2}}\left(g_{n_{i_{2}}}\right) \cdots \Delta^{\beta_{p}}\left(g_{n_{i_{p}}}\right), \quad \sum_{1 \leq s \leq p} \beta_{s}=\alpha
$$

So, to prove 1), it suffices to show that each of these products is in $I_{N}$. This follows from (2.9.1) and the assumption on $\mathcal{F}$. The proof for 2) is similar. 
Remark 2.12. The proof of Theorem 2.9, and also that of Remark 2.11, rely strongly on the fact that Tay $: B \rightarrow B[U]$, defined on the polynomial ring $B=S[X]$ by setting $\operatorname{Tay}(X)=X+U$, is an $S$-algebra homomorphism. In fact the proof of the Theorem reduces to showing that $\Delta^{\alpha}\left(H_{N}\right) \in I_{N-\alpha}$ (that $\Delta^{\alpha}\left(H_{N}\right) \in I_{N-\alpha}$ in the case of Remark 2.11), where $H_{N}=g_{n_{i_{1}}} \cdot g_{n_{i_{2}}} \cdots g_{n_{i_{p}}}$ is a product of elements in a finite set of generators $\mathcal{F}$.

An interesting, and different, $S$-algebra homomorphism is

$$
\operatorname{Tay}_{X}: B \rightarrow B[U],
$$

defined by setting $\operatorname{Tay}_{X}(X)=X+X U$. In this case

$$
\operatorname{Tay}_{X}\left(F(X)=\sum_{\alpha \geq 0} X^{\alpha} \Delta^{\alpha}(f(X)) U^{\alpha} .\right.
$$

Consider a finite set $\mathcal{F}=\left\{g_{n_{i}} \cdot W^{n_{i}}, n_{i}>0,1 \leq i \leq m\right\}$ such that:

a) For any $1 \leq i \leq m$, and any $n_{i}^{\prime}, 0<n_{i}^{\prime} \leq n_{i}: g_{n_{i}} \cdot W^{n_{i}^{\prime}} \in \mathcal{F}$.

b) For any $1 \leq i \leq m$, and for any index $0 \leq \alpha$ : $X^{\alpha} \Delta^{\alpha}\left(g_{n_{i}}\right) \cdot W^{n_{i}} \in \mathcal{F}$.

As each $g_{n_{i}}$ is polynomial on $X, X^{\alpha} \Delta^{\alpha}\left(g_{n}\right)=0$ for $\alpha$ big enough, so $\mathcal{F}$ is in fact finite; and any finite set of generators can be extended to one with these properties.

The same argument used above show that the Rees algebra generated by $\mathcal{F}$ fulfills:

$$
X^{\alpha} \Delta^{\alpha}\left(I_{n}\right) \subset I_{n}
$$

Rees algebras with this property are considered in toric geometry. They are also characterized by the fact that if $f(X)=\sum s_{r} X^{r}(\in S[X])$ is in $I_{n}$, then each $s_{r} X^{r} \in I_{n}$.

Also Theorem 2.9, as stated, can be formulated in terms of differentials with logarithmic poles.

\section{Differential algebras on smooth schemes}

3.1. A sequence of coherent ideals on a scheme $Z$, say $\left\{I_{n}\right\}_{n \in \mathbb{N}}$, such that $I_{0}=\mathcal{O}_{Z}$, and $I_{k} \cdot I_{s} \subset I_{k+s}$, defines a graded sheaf of algebras $\bigoplus_{n \geq 0} I_{n} \cdot W^{n} \subset$ $\mathcal{O}_{Z}[W]$.

We say that this algebra is a Rees algebra if there is an open covering of $Z$ by affine open sets $\left\{U_{i}\right\}$, so that

$$
\bigoplus_{n} I_{n}\left(U_{i}\right) \cdot W^{n} \subset \mathcal{O}_{Z}\left(U_{i}\right)[W]
$$

is a finitely generated $\mathcal{O}_{Z}\left(U_{i}\right)$-algebra. 
In what follows $Z$ will denote a smooth scheme of a field $k$, and $\operatorname{Diff}_{k}^{r}(Z)$, or simply Diff ${ }_{k}^{r}$, the locally free sheaf of $k$-linear differential operators of order at most $r$.

Definition 3.2. We say that a Rees algebra defined by $\left\{I_{n}\right\}_{n \in \mathbb{N}}$ is a Diffalgebra relative to the field $k$, if:

i) $I_{n} \supset I_{n+1}$.

ii) There is open covering of $Z$ by affine open sets $\left\{U_{i}\right\}$, and for any $D \in \operatorname{Diff}^{(r)}\left(U_{i}\right)$, and any $h \in I_{n}\left(U_{i}\right)$, then $D(h) \in I_{n-r}\left(U_{i}\right)$, provided $n \geq r$.

Due to the local nature of the definition, we reformulate it in terms of smooth $k$-algebras.

Definition 3.3. In what follows $R$ will denote a smooth algebra over a field, or a localization of such algebra on a closed point (a regular local ring). A Rees algebra is defined by a sequences of ideals $\left\{I_{k}\right\}_{k \in \mathbb{N}}$ such that:

1) $I_{0}=R$, and $I_{k} \cdot I_{s} \subset I_{k+s}$.

2) $\bigoplus I_{k} \cdot W^{k}$ is a finitely generated $R$-algebra.

We shall say that the Rees algebra is a Diff-algebra relative to $k$, if

3) $I_{n} \supset I_{n+1}$, and

4) given $D \in \operatorname{Diff}_{k}^{(r)}(R)$, then $D\left(I_{n}\right) \subset I_{n-r}$.

We now show that any Rees algebra extends to a smallest Diff-algebra (i.e. included in any other Diff-algebra containing it).

Theorem 3.4. Assume that $\mathcal{G}=\bigoplus I_{k} \cdot W^{k}$ is a Rees algebra over a smooth scheme $Z$. Then there is a natural and smallest extension of it, say $\mathcal{G} \subset$ $G(\mathcal{G})$, where $G(\mathcal{G})$ is a Diff-algebra relative to the field $k$.

Proof. The problem is local, so we will assume that $R$ is a local ring at a closed point of $Z$, and show that a finitely generated sub-algebra of $R[W]$ extends, by successive applications of differential operators, to a finitely generated algebra.

We will argue in steps. Assume that the local ring $R$ is of dimension 1, and let $x$ denote a parameter. Set Tay $: \widehat{R} \rightarrow \widehat{R}[[U]]$ the k-algebra morphism at the completion defined by setting $\operatorname{Tay}(x)=x+U$. Here $\widehat{R}=k^{\prime}[[x]]$ is a ring of formal power series over a finite extension $k^{\prime}$ of $k$,

$$
\operatorname{Tay}(f)=\sum \Delta^{r}(f) U^{r},
$$

and each

$$
\Delta^{r}: k^{\prime}[[x]] \rightarrow k^{\prime}[[x]]
$$

maps the sub-ring $R$ into $R$, defining $\Delta^{r}: R \rightarrow R$ (see [13, Th. 99, p. 228]). So Tay $: \widehat{R} \rightarrow \widehat{R}[[U]]$ induces by restriction Tay $: R \rightarrow R[[U]]$ (see [13]). 
For any $f \in R$ set

$$
\operatorname{Tay}(f)=\sum_{r \geq 0} \Delta^{r}(f) U^{r}(\in R[[U]]) .
$$

The operators $\Delta^{r}$ are a basis of the $k$-linear differential operators on $R$.

The same argument used in Theorem 2.9 shows that if $\bigoplus I_{k} \cdot W^{k}$ is generated by $\mathcal{F}=\left\{g_{n_{i}} \cdot W^{n_{i}} \mid n_{i}>0,1 \leq i \leq m\right\}$, then

$$
\mathcal{F}^{\prime}=\left\{\Delta^{r}\left(g_{n_{i}}\right) \cdot W^{n_{i}^{\prime}-r} \mid g_{n_{i}} \cdot W^{n_{i}} \in \mathcal{F}, \text { and } 0 \leq r<n_{i}^{\prime} \leq n_{i}\right\}
$$

generates the smallest extension to a Diff-algebra.

Let now $R$ be a localization of an arbitrary smooth algebra at a closed point, and fix a regular system of parameters $\left\{x_{1}, \ldots, x_{n}\right\}$. Define

$$
\text { Tay }: \widehat{R} \rightarrow \widehat{R}\left[\left[U_{1}, \ldots, U_{n}\right]\right]
$$

as the continuous morphisms of algebras defined by setting Tay $\left(x_{i}\right)=x_{i}+U_{i}$. So for any $h \in \widehat{R}$ set:

$$
\operatorname{Tay}(h)=\sum_{\alpha \in(\mathbb{N})^{n}} \Delta^{\alpha}(h) U^{\alpha}
$$

This morphism defines, by restriction, Tay $: R \rightarrow R\left[\left[U_{1}, \ldots, U_{n}\right]\right]$ (see [13, Theorem 99, p. 228]). Set, for $g \in R$ :

$$
\operatorname{Tay}(g)=\sum_{\alpha \in(\mathbb{N})^{n}} \Delta^{\alpha}(g) U^{\alpha} .
$$

Here $\left\{\Delta^{\alpha}\left|\alpha \in(\mathbb{N})^{n}, 0 \leq\right| \alpha \mid \leq n\right\}$ is a basis of the free $R$-module $\operatorname{Diff}^{n}(R)$, and in order to show that a Rees algebra $\bigoplus I_{k} \cdot W^{k}$ is a Diffalgebra, it suffices to check that given $g \in I_{m}$ :

$$
\Delta^{\alpha}(g) \in I_{m-|\alpha|}
$$

Note that $\Delta^{\alpha} \Delta^{\alpha^{\prime}}=\Delta^{\alpha^{\prime}} \Delta^{\alpha}$. Define, for each index $i_{0}, 1 \leq i_{0} \leq n$ :

$$
\operatorname{Tay}_{i_{0}}: R \rightarrow R\left[\left[U_{i_{0}}\right]\right],
$$

$\operatorname{Tay}_{i_{0}}\left(x_{j}\right)=x_{j}$ for $i \neq j$, and $\operatorname{Tay}_{i_{0}}\left(x_{i_{0}}\right)=x_{i_{0}}+U_{i_{0}}$. So

$$
\operatorname{Tay}_{i_{0}}(g)=\sum_{\alpha \in \mathbb{N}^{n}} \Delta_{i_{0}}^{\alpha}(g) U^{\alpha},
$$

is defined in terms of the differential operators $\Delta_{i_{0}}^{\alpha}$. 
For any $\alpha=\left(\alpha_{1}, \alpha_{2}, \ldots, \alpha_{n}\right) \in(\mathbb{N})^{n}$ :

$$
\Delta^{\alpha}=\Delta_{1}^{\alpha_{1}} \cdots \Delta_{n}^{\alpha_{n}}
$$

is a composition of partial operators defined above. And $\bigoplus I_{k} \cdot W^{k}$ is a Diffalgebra if the requirement in (3.4.1) holds for each of these partial differential operators.

So again, the arguments in Theorem 2.9 ensure that if $\bigoplus I_{k} \cdot W^{k}$ is generated by

$$
\mathcal{F}=\left\{g_{n_{i}} \cdot W^{n_{i}} \mid n_{i}>0,1 \leq i \leq m\right\}
$$

then

$$
\mathcal{F}^{\prime}=\left\{\Delta^{\alpha}\left(g_{n_{i}}\right) \cdot W^{n_{i}^{\prime}-\alpha} \mid g_{n_{i}} \cdot W^{n_{i}} \in \mathcal{F}, \alpha \in(\mathbb{N})^{n}, \text { and } 0 \leq|\alpha|<n_{i}^{\prime} \leq n_{i}\right\}
$$

generates the smallest extension of $\bigoplus I_{k} \cdot W^{k}$ to a Diff-algebra relative to the field $k$.

Remark 3.5. In the previous discussion we reduce the proof of the Theorem to the case of one variable, and we make use of Theorem 2.9. There are interesting variations in the one variable case discussed in Remark 2.12, of particular interest is that of differentials with logarithmic poles. Such is the case when we fix an integer $s, 1 \leq s \leq n$, and consider, for each index $1 \leq i_{0} \leq s$, the modified function:

$$
\operatorname{Tay}_{x_{i_{0}}}: R \rightarrow R\left[\left[U_{i_{0}}\right]\right]
$$

(defined by $\operatorname{Tay}_{x_{i_{0}}}\left(x_{j}\right)=x_{j}$ for $i \neq j$ and $\operatorname{Tay}_{x_{i_{0}}}\left(x_{i_{0}}\right)=x_{i_{0}}+x_{i_{0}} U_{i_{0}}$ ); and the usual Tay $_{j_{0}}: R \rightarrow R\left[\left[U_{i_{0}}\right]\right]$ for $s+1 \leq j_{0} \leq n$.

There is an natural analog of Diff-algebras with Rees algebras which are closed by differential operators with logarithmic poles. This follows from Remark 2.12, and it is simple to extend the outcome of (3.4.2) to this context.

Corollary 3.6. Given inclusions of Rees algebras, say

$$
\mathcal{G}=\bigoplus I_{n} \cdot W^{n} \subset \mathcal{G}^{\prime}=\bigoplus I_{n}^{\prime} \cdot W^{n} \subset G(\mathcal{G})=\bigoplus I_{n}^{\prime \prime} \cdot W^{n}
$$

where $G(\mathcal{G})$ is the Diff-algebra spanned by $\mathcal{G}$, then $G(\mathcal{G})$ is also the Diffalgebra spanned by $\mathcal{G}^{\prime}$.

3.7. Fix now a smooth morphism of smooth schemes, say $Z \rightarrow Z^{\prime}$. Let $\operatorname{Diff}_{Z^{\prime}}^{r}(Z)$, or simply $\operatorname{Diff}_{Z^{\prime}}^{r}$ denote the locally free sheaf of relative differential operators of order $r$. 
We say that the Rees algebra $\bigoplus I_{k} \cdot W^{k}$ over $Z$ (see 3.1 ) is a Diff-algebra relative to $Z^{\prime}$, if conditions in Definition 3.2 hold, where we now require that $D \in \operatorname{Diff}_{Z^{\prime}}^{r}\left(U_{i}\right)$ in (ii).

Since $\operatorname{Diff}_{Z^{\prime}}^{r}(Z) \subset \operatorname{Diff}_{k}^{r}(Z)$ it follows that any Diff-algebra relative to $k$ is also relative to $Z^{\prime}$.

Theorem 3.4 has a natural formulation for the case of Diff-algebras relative to $Z^{\prime}$. Given an ideal $I \subset \mathcal{O}_{Z}$, and a smooth morphism $Z \rightarrow Z^{\prime}$, we define an extension of ideals $I \subset \operatorname{Diff}_{Z^{\prime}}^{r}(I)$,

$$
\operatorname{Diff}_{Z^{\prime}}^{r}(I)(U)=\left\langle D(f) \mid f \in I(U), D \in \operatorname{Diff}_{Z^{\prime}}^{r}(U)\right\rangle
$$

for each open $U$ in $Z$.

Since $\operatorname{Diff}_{Z^{\prime}}^{r} \subset \operatorname{Diff}_{Z^{\prime}}^{r+1}$, clearly $\operatorname{Diff}_{Z^{\prime}}^{r}(I) \subset \operatorname{Diff}_{Z^{\prime}}^{r+1}(I)$ for $r \geq 0$.

Note finally that a Rees algebra $\bigoplus I_{k} \cdot W^{k}$ over $Z(3.1)$ is a Diff-algebra relative to $Z^{\prime}$, if and only if, for any positive integers $r \leq n, \operatorname{Diff}_{Z^{\prime}}^{r}\left(I_{n}\right) \subset$ $I_{n-r}$. In particular, for $Z^{\prime}=\operatorname{Spec}(k)$, condition ii) in Definition 3.2 can be reformulated as:

ii') $\operatorname{Diff}_{k}^{r}\left(I_{n}\right) \subset I_{n-r}$.

\section{Differential algebras and singular locus}

4.1. The notion Diff-algebra relative to a field $k$, on a smooth $k$-scheme $Z$, is closely related to the notion of order at the local regular rings of $Z$. Recall that the order of a non-zero ideal $I$ at a local regular $\operatorname{ring}(R, M)$ is the biggest integer $b$ such that $I \subset M^{b}$.

If $I \subset \mathcal{O}_{Z}$ is a sheaf of ideals, $V\left(\operatorname{Diff}_{k}^{b-1}(I)\right)$ is the closed set of points of $Z$ where the ideal has order at least $b$. We analyze this fact locally at a closed point $x$.

Let $\left\{x_{1}, \ldots, x_{n}\right\}$ be a regular system of parameters at $\mathcal{O}_{Z, x}$, and consider the differential operators $\Delta^{\alpha}$, defined on $\mathcal{O}_{Z, x}$ in terms of these parameters, as in the Theorem 3.4. So at $x$,

$$
\left(\operatorname{Diff}_{k}^{b-1}(I)\right)_{x}=\left\langle\Delta^{\alpha}(f)|f \in I, 0 \leq| \alpha \mid \leq b-1\right\rangle .
$$

One can now check at $\mathcal{O}_{Z, x}$, or at the ring of formal power series $\widehat{\mathcal{O}}_{Z, x}$, that $\operatorname{Diff}_{k}^{b-1}(I)$ is a proper ideal if and only if $I$ has order at least $b$ at the local ring.

The operators $\Delta^{\alpha}$ are defined globally at a suitable neighborhood $U$ of $x$. So if $\bigoplus I_{n} \cdot W^{n} \subset O_{Z}[W]$ is a Diff-algebra relative to the field $k$ and $x \in Z$ is a closed point, the Diff-algebra defined by localization, say $\bigoplus\left(I_{n}\right)_{x} W^{n} \subset O_{Z, x}[W]$, is properly included in $\mathcal{O}_{Z, x}[W]$, if and only if, for each index $m \in \mathbb{N}$, the ideal $\left(I_{m}\right)_{x}$ has order at least $m$ at the local regular $\operatorname{ring} \mathcal{O}_{Z, x}$. 
Definition 4.2. The singular locus of a Rees algebra $\mathcal{G}=\bigoplus I_{n} \cdot W^{n} \subset$ $O_{Z}[W]$, will be

$$
\operatorname{Sing}(\mathcal{G})=\cap_{r \geq 1} V\left(\operatorname{Diff}_{k}^{r-1}\left(I_{r}\right)\right)(\subset Z) .
$$

It is the set of points $x \in Z$ for which all $\left(I_{r}\right)_{x}$ have order at least $r$ (at $\mathcal{O}_{Z, x}$ ).

Remark 4.3. Assume that $f \in\left(I_{r}\right)_{x}$ has order $r$ at $\mathcal{O}_{Z, x}$. Then, locally at $x, \operatorname{Sing}(\mathcal{G})$ is included in the set of points of multiplicity $r$ (or say, $r$-fold points) of the hypersurface $V(\langle f\rangle)$.

In fact $\operatorname{Diff}_{k}^{r-1}(f) \subset \operatorname{Diff}_{k}^{r-1}\left(I_{r}\right)$, and the closed set defined by the first ideal is that of points of multiplicity $r$.

Proposition 4.4. 1) If $\mathcal{G}=\bigoplus I_{n} \cdot W^{n}$ and $\mathcal{G}^{\prime}=\bigoplus I_{n}^{\prime} \cdot W^{n}$ are Rees algebras with the same integral closure (e.g. if $\mathcal{G} \subset \mathcal{G}^{\prime}$ is a finite extension), then

$$
\operatorname{Sing}(\mathcal{G})=\operatorname{Sing}\left(\mathcal{G}^{\prime}\right)
$$

2) If $\mathcal{G}$ is a Rees algebra generated over $\mathcal{O}_{Z}$ by $\mathcal{F}=\left\{g_{n_{i}} \cdot W^{n_{i}}, n_{i}>0,1 \leq\right.$ $i \leq m\}$, then

$$
\operatorname{Sing}(\mathcal{G})=\cap V\left(\operatorname{Diff}^{n_{i}-1}\left(\left\langle g_{n_{i}}\right\rangle\right)\right) .
$$

3) Let $\mathcal{G}^{\prime \prime}=\bigoplus I_{n}^{\prime \prime} \cdot W^{n}$ be the extension of $\mathcal{G}$ to a Diff-algebra relative to $k$, as defined in Theorem 3.4, then $\operatorname{Sing}(\mathcal{G})=\operatorname{Sing}\left(\mathcal{G}^{\prime \prime}\right)$.

4) For any Diff-algebra $\mathcal{G}^{\prime \prime}=\bigoplus I_{n}^{\prime \prime} W^{n}, \operatorname{Sing}\left(\mathcal{G}^{\prime \prime}\right)=V\left(I_{1}^{\prime \prime}\right)$.

5) Let $\mathcal{G}^{\prime \prime}=\bigoplus I_{n}^{\prime \prime} \cdot W^{n}$ be a Diff-algebra. For any positive integer $r$, $\operatorname{Sing}\left(\mathcal{G}^{\prime \prime}\right)=V\left(I_{r}^{\prime \prime}\right)$

Proof. 1) The argument in 2.3 shows that there is an index $N$, so that $\mathcal{G}$ is finite over the subring $\bigoplus I_{N}^{k} \cdot W^{N k}$, and $\mathcal{G}^{\prime}$ is finite over $\bigoplus I_{N}^{\prime k} \cdot W^{N k}$. And furthermore, $I_{N}$ and $I_{N}^{\prime}$ have the same integral closure. In these conditions $\operatorname{Sing}(\mathcal{G})$ is the set of points $x \in Z$ such that $I_{N}$ has order at least $N$ at $\mathcal{O}_{Z, x}$, and similarly, $\operatorname{Sing}\left(\mathcal{G}^{\prime}\right)$ is the set of points $x \in Z$ such that $I_{N}^{\prime}$ has order at least $N$. Finally, the claim follows from the fact that the order of an ideal, at a local regular ring, is the same as the order of its integral closure ([23], Appendix 3).

2) We have formulated 2) with a global condition on $Z$, however this is always the case locally. In fact, there is a covering of $Z$ by affine open sets, so that the restriction of $\mathcal{G}$ is generated by finitely many elements. Let $U$ be such open set, so $\mathcal{G}(U)=\bigoplus I_{k}(U) \cdot W^{k}$ is generated by $\mathcal{F}=\left\{g_{n_{i}} \cdot W^{n_{i}}, n_{i}>\right.$ $0,1 \leq i \leq m\}, g_{n_{i}} \in \mathcal{O}_{Z}(U)$

The claim is that $y \in \operatorname{Sing}(\mathcal{G}) \cap U$ if and only if the order of $g_{n_{i}}$ at $\mathcal{O}_{Z, y}$ is at least $n_{i}$, for $1 \leq i \leq m$. 
The condition is clearly necessary. Conversely, if $\mathcal{G}=\bigoplus I_{n} \cdot W^{n}=$

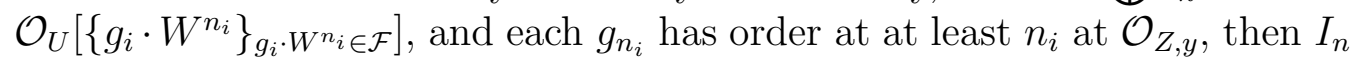
(generated by weighted homogeneous expressions on the $g_{i}$ 's) has order at least $n$ at $\mathcal{O}_{Z, y}$.

3) We argue as in 2), here we may also assume that there is $x \in U$, a regular system of parameters $\left\{x_{1}, \ldots, x_{n}\right\}$ at $x$, and differential operators $\Delta^{\alpha}$ as in Theorem 3.4, defined globally at $U$.

Formula (3.4.2) shows that the Diff-algebra $\mathcal{G}^{\prime \prime}$ in Theorem 3.4 is a finite extension of the Rees algebra defined by

$$
\mathcal{F}^{\prime}=\left\{\Delta^{\alpha}\left(g_{n_{i}}\right) \cdot W^{n_{i}-\alpha} \mid g_{n_{i}} \cdot W^{n_{i}} \in \mathcal{F}, \alpha \in(\mathbb{N})^{n}, \text { and } 0 \leq|\alpha|<n_{i}\right\}
$$

Note finally that if the order of $g_{n_{i}}$ at a local ring is $\geq n_{i}$, then the order of $\Delta^{\alpha}\left(g_{n_{i}}\right)$ is $\geq n_{i}-|\alpha|$.

4) The inclusion $\operatorname{Sing}\left(\mathcal{G}^{\prime \prime}\right) \subset V\left(I_{1}^{\prime \prime}\right)$ holds, by definition, for any Rees algebra. On the other hand, the hypothesis ensures that $\operatorname{Diff}^{r-1}\left(I_{r}^{\prime \prime}\right) \subset I_{1}^{\prime \prime}$, so $\operatorname{Sing}\left(\mathcal{G}^{\prime \prime}\right) \supset V\left(I_{1}^{\prime \prime}\right)$.

5) Follows from 4).

\section{On restrictions of differential algebras}

Proposition 5.1. Let $\mathcal{G}=\bigoplus I_{k} \cdot W^{k}$ be a Diff-algebra on a smooth scheme $V$ defined by ideals $I_{k} \subset \mathcal{O}_{V}$.

A) If $V^{\prime} \subset V$ is a closed and smooth sub-scheme, the restriction of $\mathcal{G}$ to $V^{\prime}$, say

$$
\mathcal{G}^{\prime}=\bigoplus I_{k} \mathcal{O}_{V^{\prime}} \cdot W^{k}
$$

is a Diff-algebra on $V^{\prime}$.

B) If $V^{\prime \prime} \rightarrow V$ is a smooth morphism, then the natural extension, say

$$
\mathcal{G}^{\prime \prime}=\bigoplus I_{k} \mathcal{O}_{V^{\prime \prime}} \cdot W^{k}
$$

is a Diff-algebra on $V^{\prime \prime}$.

Proof. It is clear that both $\mathcal{G}^{\prime}$ and $\mathcal{G}^{\prime \prime}$ are Rees algebras (3.1). We will show that conditions (i) and (ii) in Definition 3.2 hold.

It suffices to prove both results locally at closed points, say $x \in \operatorname{Sing}(\mathcal{G})$. Set $\mathcal{G}_{x}=\bigoplus\left(I_{k}\right)_{x} \cdot W^{k}$ where now each $\left(I_{k}\right)_{x}$ is an ideal in $\mathcal{O}_{V, x}$. We may also replace the local ring by its completion. 
A) Fix a closed point $x \in V^{\prime} \subset V$ and a local regular system of parameters, say

$$
\left\{x_{1}, \ldots, x_{h}, x_{h+1}, \ldots x_{d}\right\}
$$

at $\mathcal{O}_{V, x}$, such that $V^{\prime}$ is locally defined by the ideal $<x_{1}, \ldots, x_{h}>$. Set

$$
\widehat{\mathcal{O}}_{V, x}=k^{\prime}\left[\left[x_{1}, \ldots, x_{h}, x_{h+1}, \ldots x_{d}\right]\right],
$$

where $k^{\prime}$ is a finite extension of $k$. For each multi-index $\alpha=\left(\alpha_{1}, \ldots, \alpha_{d}\right) \in \mathbb{N}^{d}$,

$$
\Delta^{\alpha}=\Delta^{\alpha^{(1)}} \Delta^{\alpha^{(2)}}
$$

where $\alpha^{(1)}=\left(\alpha_{1}, \ldots, \alpha_{h}\right) \in \mathbb{N}^{h}$, and $\alpha^{(2)}=\left(\alpha_{h+1}, \ldots, \alpha_{d}\right) \in \mathbb{N}^{d-h}$.

Express an element $f_{n} \in I_{n}$ as

$$
f_{n}=\sum_{\alpha^{(1)} \in(\mathbb{N})^{h}} x_{1}^{\alpha_{1}} \cdots x_{h}^{\alpha_{h}} a_{\alpha^{(1)}},
$$

$a_{\alpha^{(1)}} \in k^{\prime}\left[\left[x_{h+1}, \ldots x_{d}\right]\right]$.

If $\left|\alpha^{(1)}\right|=\alpha_{1}+\cdots+\alpha_{h} \leq n$, then $a_{\alpha^{(1)}} \cdot W^{n-\left|\alpha^{(1)}\right|}$ is the class of $\Delta^{\alpha^{(1)}}\left(f_{n}\right)$. $W^{n-\left|\alpha^{(1)}\right|}$ in $\widehat{\mathcal{O}}_{V^{\prime}, x}[W]$. So it is an element in the restricted algebra. Similarly, if $\left|\alpha^{(1)}\right|+\left|\alpha^{(2)}\right| \leq n$,

$$
\Delta^{\alpha^{(2)}} a_{\alpha^{(1)}} \cdot W^{n-\left|\alpha^{(1)}\right|-\left|\alpha^{(2)}\right|}
$$

is the class of the element $\left(\Delta^{\alpha^{(2)}} \Delta^{\alpha^{(1)}}\right)\left(f_{n}\right) \cdot W^{n-\left|\alpha^{(1)}\right|-\left|\alpha^{(2)}\right|}$ in $\widehat{\mathcal{O}}_{V^{\prime}, x}[W]$.

For each index $m \geq 1, I_{m} \mathcal{O}_{V^{\prime}} \cdot W^{m}$ is defined by the coefficient $a_{0} \cdot W^{m}$ $\left(0 \in(\mathbb{N})^{h}\right)$, for each $f_{m} \cdot W^{m} \in I_{m} \cdot W^{m}$. Conditions (i) and (ii) in 3.2 are now easy to check.

For our further discussion we point out that $I_{m} \mathcal{O}_{V^{\prime}} W^{m}$ also contains all coefficients $a_{\alpha^{(1)}} \cdot W^{n-\left|\alpha^{(1)}\right|}$ of $f \cdot W^{n} \in I_{n} \cdot W^{n}$, and $n-\left|\alpha^{(1)}\right|=m$.

B) Fix a point $x^{\prime} \in V^{\prime \prime}$ mapping to $x \in V$. The completion of $\mathcal{O}_{V^{\prime \prime}, x^{\prime}}$ contains that of $\mathcal{O}_{V, x}$, say

$$
\widehat{\mathcal{O}}_{V, x}=k^{\prime}\left[\left[x_{1}, \ldots, x_{d}\right]\right] \subset \widehat{\mathcal{O}}_{V^{\prime \prime}, x^{\prime}}=k^{\prime}\left[\left[x_{1}, \ldots, x_{d}, x_{d+1}, \ldots x_{e}\right]\right] .
$$

Each ideal $I_{n}$ in $k^{\prime}\left[\left[x_{1}, \ldots, x_{d}\right]\right]$ extends to $I_{n} \cdot k^{\prime}\left[\left[x_{1}, \ldots, x_{d}, x_{d+1}, \ldots x_{e}\right]\right]$; and the claim is that the extended algebra is a Diff-algebra. The statement follows easily in this case, for example by formula (3.4.2), which expresses generators of the Diff-algebra in terms of generators of the Rees algebra.

Definition 5.2. Fix $\mathcal{G}=\bigoplus I_{k} \cdot W^{k}$, a Rees algebra on $V$, and let $V \stackrel{\pi}{\longleftarrow} V^{\prime}$ be a morphism of smooth schemes. We define the total transform of $\mathcal{G}$ to be

$$
\pi^{-1}(\mathcal{G})=\bigoplus I_{k} \mathcal{O}_{V^{\prime}} \cdot W^{k}
$$

Namely the Rees algebra defined by the total transforms of the ideals $I_{n}$, $n \geq 0$. 
Note that the restriction in A) and the natural extension in B), are particular examples of total transforms.

Lemma 5.3. Let $\mathcal{G}=\bigoplus I_{k} \cdot W^{k}\left(\subset \mathcal{O}_{V}[W]\right)$ be a Rees algebra generated by a finite set $\mathcal{F}=\left\{g_{N_{1}} \cdot W^{N_{1}}, \ldots, g_{N_{s}} \cdot W^{N_{s}}\right\}$, and let $V \stackrel{\pi}{\longleftarrow} V^{\prime}$ be a morphism of smooth schemes. Then $\pi^{-1}(\mathcal{G})$ is also generated by $\mathcal{F}$.

Proof. Since any element of $I_{M}$ is a weighted homogeneous polynomial of degree $M$ in elements of $\mathcal{F}$, the total transform of the ideal is also generated by elements that are weighted homogeneous in the same set $\mathcal{F}$.

In particular:

A) the restriction of $\mathcal{G}$ to $V^{\prime}(\subset V)$ is generated by $\left\{\bar{g}_{N_{1}} \cdot W^{N_{1}}, \ldots, \bar{g}_{N_{s}}\right.$. $\left.W^{N_{s}}\right\}$, where each $\bar{g}_{N_{i}}$ is the restriction of $g_{N_{i}}$ to $V^{\prime}$.

B) If $V^{\prime \prime} \rightarrow V$ is a smooth morphism, the total transform of $\bigoplus I_{k} \cdot W^{k}$ to $V^{\prime \prime}$ is generated by $\left\{g_{N_{1}} \cdot W^{N_{1}}, \ldots, g_{N_{s}} \cdot W^{N_{s}}\right\}$.

Theorem 5.4. Let $V^{\prime} \stackrel{\pi}{\longrightarrow} V$ be a morphism of smooth schemes, then:

i) if $\mathcal{G}$ is a Diff-algebra on $V$, the total transform $\pi^{-1}(\mathcal{G})$ is a Diff-algebra on $V^{\prime}$.

ii) $\operatorname{Sing}\left(\pi^{-1}(\mathcal{G})\right)=\pi^{-1}(\operatorname{Sing}(\mathcal{G}))$.

Proof. Since $V^{\prime} \stackrel{\pi}{\longrightarrow} V$ is of finite type, it can be expressed locally in the form $V^{\prime} \subset V^{\prime \prime} \stackrel{\beta}{\longrightarrow} V$, where $\beta$ is smooth. So Proposition 5.1 proves (i).

Fix a closed point $x \in \operatorname{Sing}\left(\pi^{-1}(\mathcal{G})\right)$. Since $\operatorname{Sing}(\mathcal{G})=V\left(I_{n}\right)$ for all $n \geq 1$ (see 4.4), it follows that $\pi(x) \in \operatorname{Sing}(\mathcal{G})$. On the other hand, if $\pi(x) \in \operatorname{Sing}(\mathcal{G})$, the order of $I_{n}$ is at least $n$ at $\mathcal{O}_{V, \pi(x)}$, for each $n \geq 1$; so the same holds at $\mathcal{O}_{V^{\prime}, x}$. This proves (ii).

\section{On differential algebras and integral closures}

6.1. Fix a Rees algebra $\mathcal{G}=\bigoplus I_{k} \cdot W^{k}\left(\subset \mathcal{O}_{V}[W]\right)$, and a point $x \in \operatorname{Sing}(\mathcal{G})$. Let $Z$ be a smooth sub-scheme containing $x$, and let $x_{1}, \ldots, x_{h}$ be part of a regular system of parameters at $\mathcal{O}_{V, x}$ so that $\left\langle x_{1}, \ldots, x_{h}\right\rangle$ is the ideal defining $Z$ locally at $x$. We define now a graded algebra over the completion, namely in $\widehat{\mathcal{O}}_{Z, x}[W]$.

This new graded algebra will be defined in terms of the (local) inclusion $Z \subset V$, and the retraction $V \rightarrow Z$ defined locally at $x$ (see 6.3).

Extend $\left\{x_{1}, \ldots, x_{h}\right\}$ to a regular system of parameters of the local ring $\mathcal{O}_{V, x}$, say $\left\{x_{1}, \ldots, x_{h}, x_{h+1}, \ldots x_{d}\right\} . \widehat{\mathcal{O}}_{V, x}$ is a ring of formal power series, say $k^{\prime}\left[\left[x_{1}, \ldots, x_{h}, x_{h+1}, \ldots x_{d}\right]\right]$, and $\widehat{\mathcal{O}}_{V^{\prime}, x}$ is $k^{\prime}\left[\left[x_{h+1}, \ldots x_{d}\right]\right]$. The local retraction is defined by $k^{\prime}\left[\left[x_{h+1}, \ldots x_{d}\right]\right] \subset k^{\prime}\left[\left[x_{1}, \ldots, x_{h}, x_{h+1}, \ldots x_{d}\right]\right]$. 
Set, as usual, $\mathcal{G}_{x}=\bigoplus I_{k} \cdot W^{k}\left(\subset \mathcal{O}_{V, x}[W]\right)$, which also extends to a Rees algebra over $\widehat{\mathcal{O}}_{V, x}$. Express an element $f_{n} \in I_{n}$ as

$$
f_{n}=\sum_{\alpha^{(1)} \in(\mathbb{N})^{h}} x_{1}^{\alpha_{1}} \cdots x_{h}^{\alpha_{h}} a_{\alpha^{(1)}}, \quad a_{\alpha^{(1)}} \in k^{\prime}\left[\left[x_{h+1}, \ldots x_{d}\right]\right] .
$$

For any such $f_{n} \cdot W^{n}$, consider the set $\left\{a_{\alpha^{(1)}} \cdot W^{n-\left|\alpha^{(1)}\right|}|0 \leq| \alpha^{(1)} \mid<n\right\}$, which we call the coefficients of $f_{n} \cdot W^{n}$. So the coefficients of $f_{n} \cdot W^{n}$ is a finite set, defined in terms of a regular system of parameters, and the weight of each coefficient depends on the index $n$.

Claim: As $f_{n} \cdot W^{n}$ varies on the Rees algebra $\mathcal{G}_{x}$, the coefficients of $f_{n} \cdot W^{n}$ generate a Rees algebra, say $\operatorname{Coeff}(\mathcal{G})_{x}$, in $k^{\prime}\left[\left[x_{h+1}, \ldots x_{d}\right]\right][W]$.

The claim here is that the graded algebra $\operatorname{Coeff}(\mathcal{G})_{x}$ is a finitely generated sub-algebra of $k^{\prime}\left[\left[x_{h+1}, \ldots x_{d}\right]\right][W]$.

Assume that $\mathcal{F}=\left\{g_{N_{1}} \cdot W^{N_{1}}, \ldots, g_{N_{s}} \cdot W^{N_{s}}\right\}$ generate $\mathcal{G}_{x}$. Express, for $1 \leq i \leq s$

$$
g_{N_{i}}=\sum_{\alpha \in(\mathbb{N})^{h}} x_{1}^{\alpha_{1}} \cdots x_{h}^{\alpha_{h}} a_{\alpha}^{(i)} \quad a_{\alpha} \in k^{\prime}\left[\left[x_{h+1}, \ldots x_{d}\right]\right] .
$$

We search for a finite set of coefficients, that $\operatorname{span} \operatorname{Coeff}(\mathcal{G})_{x}$. A first candidate would be

$$
\mathcal{F}_{1}^{\prime}=\left\{a_{\alpha}^{(i)} \cdot W^{N_{i}-|\alpha|}|0 \leq| \alpha \mid<N_{i}, 1 \leq i \leq s\right\} .
$$

Consider the product of two elements in $\mathcal{F}$, say $g_{N_{i}} \cdot W^{N_{i}} \cdot g_{N_{j}} \cdot W^{N_{j}}=f_{n} \cdot W^{n}$ $\left(n=N_{i}+N_{j}\right)$; and a coefficient, say $a_{\alpha^{(1)}} \cdots W^{n-\left|\alpha^{(1)}\right|}$, of $f_{n} \cdot W^{n}$.

It follows from 6.1.1 that

$$
a_{\alpha^{(1)}}=\sum_{\beta+\delta=\alpha^{(1)}} a_{\beta}^{(i)} a_{\delta}^{(j)}
$$

for $\beta, \delta$, and $\alpha^{(1)}$ in $(\mathbb{N})^{h}$. Note that we cannot extract from the previous, expressions of the form

$$
a_{\alpha^{(1)}} \cdot W^{n-\left|\alpha^{(1)}\right|}=\sum_{\beta+\delta=\alpha^{(1)}} a_{\beta}^{(i)} \cdot W^{N_{i}-|\beta|} a_{\delta}^{(j)} \cdot W^{N_{j}-|\delta|} .
$$

In fact, it can happen that $|\delta| \geq N_{j}$, and we only consider $W$ with positive exponents. In particular, the previous expression of $a_{\alpha^{(1)}} \cdot W^{n-\left|\alpha^{(1)}\right|}$ is not weighted homogeneous in $\mathcal{F}_{1}^{\prime}$, and hence not in the graded sub-algebra of $k^{\prime}\left[\left[x_{h+1}, \ldots x_{d}\right]\right][W]$ generated by $\mathcal{F}_{1}^{\prime}$. 
One way to remedy this situation is to allow $a_{\beta}^{(i)}$ to have weight $n-\left|\alpha^{(1)}\right|$ if $|\delta| \geq N_{j}$. Note that in such case

$$
n-\left|\alpha^{(1)}\right|=N_{i}-|\beta|+N_{j}-|\delta| \leq N_{i}-|\beta| .
$$

Therefore $\mathcal{F}_{1}^{\prime}$ can be enlarged to say,

$$
\mathcal{F}_{1}=\left\{a_{\alpha}^{(i)} \cdot W^{n_{i, \alpha}}|0 \leq| \alpha\left|<N_{i}, 1 \leq i \leq s, 0<n_{i, \alpha} \leq N_{i}-\right| \alpha \mid\right\},
$$

for $N_{i}$ and $\alpha$ as in $\mathcal{F}_{1}^{\prime}$; and the coefficients of $f_{n} \cdot W^{n}$ are now weighted homogeneous on $\mathcal{F}_{1}$ (i.e. are in the sub-algebra of $k^{\prime}\left[\left[x_{h+1}, \ldots x_{d}\right]\right][W]$ generated by $\left.\mathcal{F}_{1}\right)$.

The argument applied here to $g_{N_{i}} \cdot W^{N_{i}} \cdot g_{N_{j}} \cdot W^{N_{j}}$, also holds for the coefficients of any product of elements in $\mathcal{F}$, and hence for the coefficients of any homogeneous element in the algebra generated by $\mathcal{F}=$ $\left\{g_{N_{1}} \cdot W^{N_{1}}, \ldots, g_{N_{s}} \cdot W^{N_{s}}\right\}$ (i.e. for the coefficients of any homogeneous element of $\mathcal{G}_{x}$ ).

This shows that there is an inclusion of subalgebras in $k^{\prime}\left[\left[x_{h+1}, \ldots x_{d}\right]\right][W]$, say

$$
k^{\prime}\left[\left[x_{h+1}, \ldots x_{d}\right]\right]\left[\mathcal{F}_{1}^{\prime}\right] \subset \operatorname{Coeff}(\mathcal{G})_{x} \subset k^{\prime}\left[\left[x_{h+1}, \ldots x_{d}\right]\right]\left[\mathcal{F}_{1}\right] .
$$

On the other hand $k^{\prime}\left[\left[x_{h+1}, \ldots x_{d}\right]\right]\left[\mathcal{F}_{1}^{\prime}\right] \subset k^{\prime}\left[\left[x_{h+1}, \ldots x_{d}\right]\right]\left[\mathcal{F}_{1}\right]$ is a finite extension $(2.2,2))$. In particular $\operatorname{Coeff}(\mathcal{G})_{x}$ is finitely generated.

Remark 6.2. 1) $\mathcal{F}_{1}^{\prime}$ can be extended to a finite set, say $\mathcal{F}_{1}^{\prime \prime}$, of generators of $\operatorname{Coeff}(\mathcal{G})_{x}$.

2) $\operatorname{Sing}\left(k^{\prime}\left[\left[x_{h+1}, \ldots x_{d}\right]\right]\left[\mathcal{F}_{1}^{\prime}\right]\right)=\operatorname{Sing}\left(\operatorname{Coeff}(\mathcal{G})_{x}\right)(\operatorname{Prop} 4.4,(1))$.

3) $\operatorname{Sing}\left(\operatorname{Coeff}(\mathcal{G})_{x}\right)$ can be naturally identified with the intersection $Z \cap$ $\operatorname{Sing}(\mathcal{G})$ locally at the point $x$.

To check 3) note that the singular locus of $k^{\prime}\left[\left[x_{h+1}, \ldots x_{d}\right]\right]\left[\mathcal{F}_{1}^{\prime}\right]$ can be naturally identified with the intersection $Z \cap \operatorname{Sing}(\mathcal{G})$. This follows from the definition of $\mathcal{F}_{1}^{\prime}$ in (6.1.2), and the expressions in (6.1.1). Finally apply 2).

6.3. Fix, as in 6.1, an inclusion of smooth schemes $Z \subset V$, and a closed point $x \in Z$. Assume that there is a retraction say $V \rightarrow Z$ locally at $x$. Let $x_{1}, \ldots, x_{h}$ be part of a regular system of parameters at $\mathcal{O}_{V, x}$ so that $<x_{1}, \ldots, x_{h}>$ defines $Z$ at $\mathcal{O}_{V, x}$; and let $\left\{x_{h+1}, \ldots x_{d}\right\}$ be a regular system of parameters at $\mathcal{O}_{Z, x}$. The local retraction at the point $x$ defines an inclusion $\mathcal{O}_{Z, x} \subset \mathcal{O}_{V, x}$, so we may consider $\left\{x_{1}, \ldots, x_{h}, x_{h+1}, \ldots x_{d}\right\}$ as parameters at $\mathcal{O}_{V, x}$.

We may identify $\widehat{\mathcal{O}}_{V, x}$ with a ring of power series $k^{\prime}\left[\left[x_{1}, \ldots, x_{h}, x_{h+1}, \ldots x_{d}\right]\right]$, $\widehat{\mathcal{O}}_{Z, x}$ with $k^{\prime}\left[\left[x_{h+1}, \ldots x_{d}\right]\right]$; and the local retraction defines the inclusion

$$
k^{\prime}\left[\left[x_{h+1}, \ldots x_{d}\right]\right] \subset k^{\prime}\left[\left[x_{1}, \ldots, x_{h}, x_{h+1}, \ldots x_{d}\right]\right] .
$$


Given $\mathcal{G}=\bigoplus I_{k} \cdot W^{k}\left(\subset \mathcal{O}_{V}[W]\right)$, we have defined Coeff $(\mathcal{G})$ at $\widehat{\mathcal{O}}_{Z, x}[W]$. We now show that it can also be defined in $\mathcal{O}_{Z, x}[W]$, and that the definition relies on the local retraction and the local inclusion. Express an element $f_{n} \in I_{n} \widehat{\mathcal{O}}_{V, x}$ as

$$
f_{n}=\sum_{\alpha^{(1)} \in(\mathbb{N})^{h}} x_{1}^{\alpha_{1}} \cdots x_{h}^{\alpha_{h}} a_{\alpha^{(1)}},
$$

$a_{\alpha^{(1)}} \in k^{\prime}\left[\left[x_{h+1}, \ldots x_{d}\right]\right]$. For each multi-index $\alpha^{(1)}, 0 \leq\left|\alpha^{(1)}\right| \leq n$, the coefficient $a_{\alpha^{(1)}}$ can be identified with the class of $\Delta^{\alpha^{(1)}}\left(f_{n}\right)$ in $\widehat{\mathcal{O}}_{Z, x}$. However, $\Delta^{\alpha^{(1)}}$ is a differential operator, relative to the local retraction $V \rightarrow Z$, $\Delta^{\alpha^{(1)}}\left(f_{n}\right)$ is an element in $\mathcal{O}_{V, x}$, and we can therefore consider the class of this element in $\mathcal{O}_{Z, x}$.

This shows that $\operatorname{Coeff}(\mathcal{G})\left(\subset \mathcal{O}_{Z, x}[W]\right)$, is the restriction via $Z \subset V$, of the extension of $\mathcal{G}$ defined by the Diff-algebra relative to the local retraction (see 3.7). In other words, and from an algebraic point of view, Coeff $(\mathcal{G})(\subset$ $\left.\mathcal{O}_{Z, x}[W]\right)$ is defined in terms of:

i) the surjection $\mathcal{O}_{V, x} \rightarrow \mathcal{O}_{Z, x}$; and

ii) the inclusion $\mathcal{O}_{Z, x} \subset \mathcal{O}_{V, x}$ (etale locally).

This proves the following Remark in the formal case:

Remark 6.4. Consider $\mathcal{G} \subset \mathcal{O}_{V, x}[W]$, and, as before, a surjection $\widehat{\mathcal{O}}_{V, x} \rightarrow$ $\widehat{\mathcal{O}}_{Z, x}$; and an inclusion $\widehat{\mathcal{O}}_{Z, x} \subset \widehat{\mathcal{O}}_{V, x}$. Let $\left\{x_{h+1}, \ldots, x_{d}\right\}$ be a regular system of parameters at $\mathcal{O}_{Z, x}$, and assume that $\left\{x_{1}, \ldots x_{h}, x_{h+1}, \ldots, x_{d}\right\}$ and $\left\{x_{1}^{\prime}, \ldots x_{h}^{\prime}, x_{h+1}, \ldots, x_{d}\right\}$ are two extensions to regular system of parameters at $\mathcal{O}_{V, x}$, and that $\left.\left\langle x_{1}, \ldots, x_{h}\right\rangle=<x_{1}^{\prime}, \ldots, x_{h}^{\prime}\right\rangle=I(Z) \subset \mathcal{O}_{V, x}$

The same inclusion $\mathcal{O}_{Z, x} \subset \mathcal{O}_{V, x}$ can be expressed as

$$
\widehat{\mathcal{O}}_{Z, x}=k^{\prime}\left[\left[x_{h+1}, \ldots, x_{d}\right]\right] \subset k^{\prime}\left[\left[x_{1}, \ldots, x_{h}, x_{h+1}, \ldots, x_{d}\right]\right]=\widehat{\mathcal{O}}_{V, x}
$$

or as

$$
\widehat{\mathcal{O}}_{Z, x}=k^{\prime}\left[\left[x_{h+1}, \ldots, x_{d}\right]\right] \subset k^{\prime}\left[\left[x_{1}^{\prime}, \ldots, x_{h}^{\prime}, x_{h+1}, \ldots, x_{d}\right]\right]=\widehat{\mathcal{O}}_{V, x} .
$$

In both cases $\operatorname{Coeff}(\mathcal{G}) \subset \widehat{\mathcal{O}}_{Z, x}[W]$ is the same.

The discussion in 6.3 also shows that, of course, the definition of Coeff $(\mathcal{G})$ $\subset \widehat{\mathcal{O}}_{Z, x}[W]$ is independent of the coordinates we choose in the subring $\widehat{\mathcal{O}}_{Z, x}$.

6.5. Set $\mathcal{G} \subset \mathcal{O}_{V, x}[W]$ and

$$
\widehat{\mathcal{O}}_{Z, x}=k^{\prime}\left[\left[x_{h+1}, \ldots, x_{d}\right]\right] \subset k^{\prime}\left[\left[x_{1}, \ldots, x_{h}, x_{h+1}, \ldots, x_{d}\right]\right]=\widehat{\mathcal{O}}_{V, x}
$$

as above, where $<x_{1}, \ldots, x_{h}>=I(Z) \subset \widehat{\mathcal{O}}_{V, x}$. 
Note that the natural identification of $k^{\prime}\left[\left[x_{h+1}, \ldots, x_{d}\right]\right]$ with $\widehat{\mathcal{O}}_{V, x} / I(Z)$, provides an inclusion:

$$
\overline{\mathcal{G}} \subset \operatorname{Coeff}(\mathcal{G}) \subset \widehat{\mathcal{O}}_{Z, x}[W]
$$

where $\overline{\mathcal{G}} \subset\left(\widehat{\mathcal{O}}_{V, x} / I(Z)\right)[W]$ denotes the restriction. Furthermore, this inclusion is an equality if $\mathcal{G}$ is a differential algebra (see also Lemma 6.6 below).

Lemma 6.6. With the setting as above, the restriction of $G(\mathcal{G})$ to the smooth sub-scheme $Z$ is the Diff-algebra spanned by $\operatorname{Coeff}(\mathcal{G})$ (i.e. the Diff-algebra generated by $\operatorname{Coeff}(\mathcal{G})$ in $\left.\mathcal{O}_{Z, x}[W]\right)$.

Proof. The previous discussion shows that $\operatorname{Coeff}(\mathcal{G})$ is included in the restriction of $G(\mathcal{G})$, which is a Diff-algebra over $\mathcal{O}_{Z, x}$ (Proposition 5.1,A)). In particular, the Diff-algebra spanned by $\operatorname{Coeff}(G)_{x}$ is included in the restriction. The claim is that this last inclusion is an equality.

Here $G(\mathcal{G})=\bigoplus I_{k}^{\prime} \cdot W^{k}$ is the Diff-algebra generated by $\mathcal{G}$, so to prove this equality it suffices to show that given $f_{n} \in I_{n}$, and $\alpha=\left(\alpha_{1}, \ldots, \alpha_{d}\right) \in(\mathbb{N})^{d}$, $0 \leq|\alpha|<n$, the class of $\Delta^{\alpha}\left(f_{n}\right) \cdot W^{n-|\alpha|}$ in $\mathcal{O}_{Z, x}[W]$, is in the Diff-algebra generated by $\operatorname{Coeff}(\mathcal{G})$.

For this last claim we argue as in the proof of Proposition 5.1, (A), by splitting each multi-index $\alpha=\left(\alpha_{1}, \ldots, \alpha_{d}\right) \in(\mathbb{N})^{d}$ :

$$
\Delta^{\alpha}=\Delta^{\alpha^{(1)}} \Delta^{\alpha^{(2)}}
$$

where $\alpha^{(1)}=\left(\alpha_{1}, \ldots, \alpha_{h}\right)$, and $\alpha^{(2)}=\left(\alpha_{h+1}, \ldots, \alpha_{d}\right)$.

The class of $\Delta^{\alpha^{(1)}}\left(f_{n}\right) \cdot W^{n-\left|\alpha^{(1)}\right|}$ is $a_{\alpha^{(1)}} \cdot W^{n-\left|\alpha^{(1)}\right|} \in \operatorname{Coeff}(\mathcal{G})$; and that of $\Delta^{\alpha}\left(f_{n}\right) \cdot W^{n-|\alpha|}$ is $\Delta^{\alpha^{(2)}}\left(a_{\alpha^{(1)}}\right) \cdot W^{n-\left|\alpha^{(1)}\right|-\left|\alpha^{(2)}\right|}$, which is clearly in the Diffalgebra spanned by $\operatorname{Coeff}(\mathcal{G})$.

Corollary 6.7. Fix a smooth scheme $V$, a Rees algebra $\mathcal{G}$, and a closed and smooth sub-scheme $Z$ of $V$. If $G(\mathcal{G})$ denotes the Diff-algebra spanned by $\mathcal{G}$, and if $[G(\mathcal{G})]_{Z}$ denotes the restriction to $Z$, then $\operatorname{Sing}\left([G(\mathcal{G})]_{Z}\right)$, as closed set in $Z$, can be identified with $Z \cap \operatorname{Sing}(\mathcal{G})$.

This follows from Lemma 6.6 and $6.2,3)$.

Remark 6.8. Let $\mathcal{G}=\bigoplus I_{k} \cdot W^{k}\left(\subset \mathcal{O}_{V^{\prime}}[W]\right)$ be a Rees algebra on a one dimensional smooth scheme $V^{\prime}$. If we assume that some $I_{k} \neq 0$, then $\operatorname{Sing}(\mathcal{G})$ is a finite set of points.

Fix $x \in \operatorname{Sing}(\mathcal{G})$ and set $\widehat{\mathcal{O}}_{V^{\prime}, x}=k^{\prime}[[t]]$, so

$$
\mathcal{G}=\bigoplus_{r \geq 1}<t^{a_{r}}>\cdot W^{r}
$$


and $a_{r} \geq r$ for each index $r$. Define

$$
\lambda_{\mathcal{G}}=\inf _{r}\left\{\frac{a_{r}}{r}\right\}
$$

and note that $\lambda_{\mathcal{G}} \geq 1$.

Let $\left\{g_{N_{1}} \cdot W^{N_{1}}, \ldots, g_{N_{s}} \cdot W^{N_{s}}\right\}$ be a set of generator locally at a closed point $x \in \operatorname{Sing}(\mathcal{G})$. Fix any integer $M$ divisible by all $N_{i}, 1 \leq i \leq s$, then

$$
\lambda_{\mathcal{G}}=\frac{\nu\left(I_{M}\right)}{M}
$$

where $\nu\left(I_{M}\right)$ denotes the order of the ideal at $\mathcal{O}_{V^{\prime}, x}$. Let $\overline{\mathcal{G}}$ denote the integral closure of $\mathcal{G}$.

Claim 1: The integral closure of $\mathcal{G}$ is determined by the rational number $\lambda_{\mathcal{G}}$, and $\lambda_{\mathcal{G}}=\lambda_{\overline{\mathcal{G}}}$.

In fact, by usual arguments of toric geometry, we conclude that $t^{n} \cdot W^{m} \in$ $\overline{\mathcal{G}}$, if and only if $\frac{n}{m} \geq \lambda_{\mathcal{G}}$. This proves the claim.

Let $G(\mathcal{G})$ denote the Diff-algebra spanned by $\mathcal{G}$. Recall that $\operatorname{Sing}(\mathcal{G})=$ $\operatorname{Sing}(G(\mathcal{G}))$.

Claim 2: Locally at any $x \in \operatorname{Sing}(\mathcal{G})$, both $\mathcal{G}$ and $G(\mathcal{G})$ have the same integral closure.

We prove our claim by showing that $\lambda_{\mathcal{G}}=\lambda_{\mathcal{G}^{\prime}}$. To this end note that given $t^{a} \cdot W^{b} \in \mathcal{G}$, and an operator $\Delta^{j}, 0 \leq j<b$,

$$
\Delta^{j}\left(t^{a}\right) \cdot W^{b-j}=d \cdot t^{a-j} \cdot W^{b-j},
$$

where $d$ is the class of an integer in the field $k^{\prime}$. Since $a \geq b>j \geq 0$ it follows that $\frac{a-j}{b-j} \geq \frac{a}{b}$, so Claim 2 follows from Claim 1 .

6.9. The previous Remark shows that in the one dimensional case, the extension $\mathcal{G} \subset G(\mathcal{G})$ is finite, where $G(\mathcal{G})$ is the Diff-algebra spanned by $\mathcal{G}$.

In general $G(\mathcal{G})$ is not integrally closed. Consider, for example, the semi-group in $\mathbb{N} \times \mathbb{N}$ defined by the pairs $(x, y)$ such that $-2 x+y \geq 0$ and $-x+y \geq 3$. Use the previous remark to show that the set of pairs $\left\{t^{i} \cdot W^{j}\right\}$, where $(j, i)$ fulfills the previous inequalities, form a Diff-algebra $\mathcal{G}$ which is not integrally closed. In fact $t^{3} \cdot W$ is integral over $\mathcal{G}$.

6.10. Let $\mathcal{G}$ be a Rees algebra over a smooth scheme $V$, generated by $\left\{g_{N_{1}} \cdot W^{N_{1}}, \ldots, g_{N_{s}} \cdot W^{N_{s}}\right\}$, which we may assume by restriction to affine open sets. Let $M$ is a positive integer divisible by all $N_{j}, 1 \leq j \leq s$; and consider the Rees ring $\mathcal{O}_{V}\left[I_{M} \cdot W^{M}\right]$. Recall that $\mathcal{O}_{V}\left[I_{M} \cdot W^{M}\right] \subset \mathcal{G}$ is a finite extension of graded algebras, and that any Rees algebra is a finite extension of a Rees ring of an ideal (see 2.3). 
Given two Rees algebras $\mathcal{G}_{1}=\bigoplus_{r>0} I(1)_{r} \cdot W^{r}$ and $\mathcal{G}_{2}=\bigoplus_{r>0} I(2)_{r}$. $W^{r}$, there is a positive integer $M$ such that both are integral extensions of the Rees ring generated by the $M$-th term, say $\bigoplus_{k \geq 0} I(1)_{M}^{k} \cdot W^{k M}$ and $\bigoplus_{k \geq 0} I(2)_{M}^{k} \cdot W^{k M}$.

Proposition 6.11. Fix two Rees algebras $\mathcal{G}_{1}$ and $\mathcal{G}_{2}$ on a smooth scheme $V$ over a field $k$. Assume that for any morphism of regular $k$-schemes, say $V^{\prime} \stackrel{\pi}{\longrightarrow} V$, where $V^{\prime}$ is one dimensional, both pull-backs have the same integral closure (i.e. $\left.\overline{\pi^{-1}\left(\mathcal{G}_{1}\right)}=\overline{\pi^{-1}\left(\mathcal{G}_{2}\right)}\right)$. Then $\mathcal{G}_{1}$ and $\mathcal{G}_{2}$ have the same integral closure in $V$.

Proof. Choose $M$ and ideals $I(1)_{M}$ and $I(2)_{M}$ as in 6.10. We may assume here that $\pi$ is of finite type. Lemma 5.3 and the previous properties show that under the condition of the hypothesis both $I(1)_{M}$ and $I(2)_{M}$ have the same integral closure in $\mathcal{O}_{V}$ (see 2.5). In particular, $\mathcal{G}_{1}$ and $\mathcal{G}_{2}$ have the same integral closure.

Proposition 6.12. Let $\mathcal{G}_{1} \subset \mathcal{G}_{2}\left(\subset \mathcal{O}_{V}[W]\right)$ be a finite extension of Rees algebras on a smooth scheme $V$, and let $V^{\prime}$ be a smooth one dimensional subscheme in $V$. Fix $x \in V^{\prime}$ and a regular system of coordinates $\left\{x_{1}, \ldots, x_{d-1}, x_{d}\right\}$ at $\mathcal{O}_{V, x}$, so that the curve is locally defined by $\left\langle x_{1}, \ldots, x_{d-1}\right\rangle$. Then

$$
\operatorname{Coeff}\left(\mathcal{G}_{1}\right) \subset \operatorname{Coeff}\left(\mathcal{G}_{2}\right)
$$

is a finite extension in $\mathcal{O}_{V^{\prime}}[W]$.

Proof. Express any $f \in \widehat{\mathcal{O}}_{V}=k^{\prime}\left[\left[x_{1}, \ldots, x_{d-1}, x_{d}\right]\right]$ as:

$$
f=\sum_{\alpha \in(\mathbb{N})^{d-1}} x_{1}^{\alpha_{1}} \cdots x_{d-1}^{\alpha_{d-1}} a_{\alpha} \quad a_{\alpha} \in k^{\prime}\left[\left[x_{d}\right]\right] .
$$

The coefficients of $f \cdot W^{N}$ are $\left\{a_{\alpha} \cdot W^{N-|\alpha|}|0 \leq| \alpha \mid<N\right\}$, and we define

$$
s l_{V^{\prime}}\left(f \cdot W^{N}\right)=\min \left\{\frac{\nu\left(a_{\alpha}\right)}{N-|\alpha|}|0 \leq| \alpha \mid<N\right\},
$$

where $\nu\left(a_{\alpha}\right)$ denotes the order of $a_{\alpha}$ in $k^{\prime}\left[\left[x_{d}\right]\right]$. Set $\operatorname{Coeff}\left(\mathcal{G}_{1}\right)$ and $\operatorname{Coeff}\left(\mathcal{G}_{2}\right)$ in $\mathcal{O}_{V^{\prime}}[W]$, as in (6.1). Assume that $\mathcal{F}_{1}=\left\{f_{N_{1}} \cdot W^{N_{1}}, \ldots, f_{N_{s}} \cdot W^{N_{s}}\right\}$ generate $\mathcal{G}_{1}$ locally at $x$, and that $\mathcal{F}_{2}=\left\{g_{M_{1}} \cdot W^{M_{1}}, \ldots, g_{M_{t}} \cdot W^{M_{t}}\right\}$ generate $\mathcal{G}_{2}$.

The inclusion Coeff $\left(\mathcal{G}_{1}\right) \subset \operatorname{Coeff}\left(\mathcal{G}_{2}\right)$ at $\mathcal{O}_{V^{\prime}}[W]$ is clear.

Set $\operatorname{Coeff}\left(\mathcal{G}_{i}\right)=\bigoplus_{r \geq 0} J(i)_{r} W^{r}$ in $\mathcal{O}_{V^{\prime}}[W]$, for $i=1,2$. Note that $J(1)_{r}=0$ for all $r \geq 1$ iff $V^{\prime} \subset \operatorname{Sing}\left(\mathcal{G}_{1}\right)$ iff $\mathcal{G}_{1} \subset \bigoplus_{r \geq 0} P^{r} W^{r}$; where $P$ is the ideal defining the smooth sub-scheme $V^{\prime}$. Since $\mathcal{G}_{1} \subset \mathcal{G}_{2}$ is finite, it follows that also $J(2)_{r}=0$ for all $r \geq 1$. 
Assume now that some $J(1)_{r}$ is not zero for some $r>0$. The inclusion $\operatorname{Coeff}\left(\mathcal{G}_{1}\right) \subset \operatorname{Coeff}\left(\mathcal{G}_{2}\right)$ ensures that

$$
\lambda_{\text {Coeff }\left(\mathcal{G}_{1}\right)} \geq \lambda_{\text {Coeff }\left(\mathcal{G}_{2}\right)},
$$

and we shall prove the claim, in what follows, by showing that they are equal (see Remark 6.8).

Each $g_{M_{j}} \cdot W^{M_{j}}$ is integral over the localization of $\mathcal{G}_{1}$ in $\mathcal{O}_{V, x}[W]$. And this property is preserved by any change of rings. Namely, for any ring homomorphism $\phi: \mathcal{O}_{V, x} \rightarrow S, \phi\left(\mathcal{G}_{1}\right)$ is a Rees algebra in $S[W]$, and $\phi\left(g_{M_{j}}\right) \cdot W^{M_{j}}$ is integral over $\phi\left(\mathcal{G}_{1}\right)$.

Express, for any $g_{M_{j}} \cdot W^{M_{j}} \in \mathcal{F}_{2}$ :

$$
g_{M_{j}}=\sum_{\alpha \in(\mathbb{N})^{h}} x_{1}^{\alpha_{1}} \cdots x_{h}^{\alpha_{h}} a_{\alpha}^{(j)} \quad a_{\alpha} \in k^{\prime}\left[\left[x_{d}\right]\right]
$$

and set

$$
\mathcal{F}_{2}^{\prime}=\left\{a_{\alpha}^{(j)} \cdot W^{M_{j}-|\alpha|}|0 \leq| \alpha \mid<M_{j}, 1 \leq j \leq t\right\}
$$

(coefficients of all $g_{M_{j}}$ 's).

We know that $k^{\prime}\left[\left[x_{d}\right]\right]\left[\mathcal{F}_{2}^{\prime}\right] \subset \operatorname{Coeff}\left(\mathcal{G}_{2}\right)$ is a finite extension in $k^{\prime}\left[\left[x_{d}\right]\right][W]$ (see 6.1.4); in particular:

$$
\lambda_{\text {Coeff }\left(\mathcal{G}_{2}\right)}=\min \left\{\frac{\nu\left(a_{\alpha}^{(j)}\right)}{M_{j}-|\alpha|}|0 \leq| \alpha \mid<M_{j}, 1 \leq j \leq t\right\}
$$

or, equivalently:

$$
\lambda_{\text {Coeff }\left(\mathcal{G}_{2}\right)}=\min \left\{s l_{V^{\prime}}\left(g_{M_{j}}\right) \mid 1 \leq j \leq t\right\}
$$

So equality in (6.12.1) would follow if we show that $\lambda_{\text {Coeff }\left(\mathcal{G}_{1}\right)} \leq \frac{\nu\left(a_{\alpha}^{(j)}\right)}{M_{j}-|\alpha|}$ for each fraction as above.

We will assume that

$$
\frac{\nu\left(a_{\alpha}^{\left(j_{0}\right)}\right)}{M_{j_{0}}-|\alpha|}<\lambda_{\text {Coeff }\left(\mathcal{G}_{1}\right)}
$$

for some index $1 \leq j_{0} \leq t$, or equivalently, that $s l_{V^{\prime}}\left(g_{M_{j_{0}}}\right)<\lambda_{\text {Coeff }\left(\mathcal{G}_{1}\right)}$ for some index $j_{0}$, and show that in such case $g_{M_{j_{0}}} \cdot W^{M_{j_{0}}}$ is not integral over $\mathcal{G}_{1}$; which is a contradiction.

Define, as before, $s l_{V^{\prime}}\left(f_{N_{i}}\right)$ for each $f_{N_{i}} \cdot W^{N_{i}} \in \mathcal{F}_{1}$, so that

$$
\lambda_{\text {Coeff }\left(\mathcal{G}_{1}\right)}=\min \left\{s l_{V^{\prime}}\left(f_{N_{i}}\right) \mid 1 \leq i \leq s\right\} .
$$


We will show that if $s l_{V^{\prime}}\left(g_{M_{j_{0}}}\right)<\lambda_{\text {Coeff }\left(\mathcal{G}_{1}\right)}$, for some index $j_{0}$, a ring $S$ and a morphism $\phi: \widehat{\mathcal{O}}_{V}=k^{\prime}\left[\left[x_{1}, \ldots, x_{d-1}, x_{d}\right]\right] \rightarrow S$ can be defined so that $\phi\left(g_{M_{j_{0}}}\right) \cdot W^{M_{j_{0}}}$ is not integral over $\phi\left(\mathcal{G}_{1}\right)$.

Given $f=\sum_{\alpha} \lambda_{\alpha} x_{1}^{\alpha_{1}} \ldots x_{d-1}^{\alpha_{d-1}} x_{d}^{\alpha_{d}} \in k^{\prime}\left[\left[x_{1}, \ldots, x_{d-1}, x_{d}\right]\right]$, set

$$
\operatorname{Supp}(f)=\left\{\alpha \in \mathbb{N}^{d} \mid \lambda_{\alpha} \neq 0\right\} \text {. }
$$

Let $a>0$ and $b>0$ be positive integers such that

$$
\lambda=\lambda_{\text {Coeff }\left(\mathcal{G}_{1}\right)}=\frac{a}{b} .
$$

Define $l: \mathbb{R}^{d} \rightarrow \mathbb{R}, l\left(y_{1}, \ldots, y_{d}\right)=a y_{1}+a y_{2}+\cdots+a y_{d-1}+b y_{d}$, which maps $\mathbb{N}^{d}$ into $\mathbb{N}$.

It follows that for a fixed integer $N$ :

$l(N, 0, \ldots, 0,0)=l(0, N, \ldots, 0,0)=\cdots=l(0, \ldots, N, 0)=l(0, \ldots, 0, \lambda N)=a N$.

Given $\left(\alpha_{1}, \ldots, \alpha_{d-1}, s\right) \in \mathbb{N}^{d}$, if $l\left(\alpha_{1}, \alpha_{2}, \ldots, \alpha_{d-1}, s\right)<a N,|\alpha|:=\alpha_{1}+\cdots+$ $\alpha_{d-1}<N$. Furthermore:

$$
l\left(\alpha_{1}, \alpha_{2}, \ldots, \alpha_{d-1}, s\right)<a N \Leftrightarrow a|\alpha|+b s<a N \Leftrightarrow \frac{s}{N-|\alpha|}<\lambda .
$$

We show now that:

1) For each $f_{N_{i}} \cdot W^{N_{i}} \in \mathcal{F}_{1}, \operatorname{Supp}\left(f_{N_{i}}\right)$ is included in the half space $l\left(y_{1}, \ldots, y_{d}\right) \geq a N_{i}$.

2) For some $f_{N_{i}} \cdot W^{N_{i}} \in \mathcal{F}_{1}$, the intersection of $\operatorname{Supp}\left(f_{N_{i}}\right)$ with the hyperplane $l\left(y_{1}, \ldots, y_{d}\right)=a N_{i}$ is not empty.

3) For some $g_{M_{j_{0}}} \cdot W^{M_{j_{0}}} \in \mathcal{F}_{2}, \operatorname{Supp}\left(g_{M_{j_{0}}}\right)$ is not included in the half space $l\left(y_{1}, \ldots, y_{d}\right) \geq a M_{j_{0}}$.

In order to prove 1$)$ set

$$
f_{N_{i}}=\sum_{\alpha \in(\mathbb{N})^{d-1}} x_{1}^{\alpha_{1}} \cdots x_{d-1}^{\alpha_{d-1}} a_{\alpha}^{(i)} \quad a_{\alpha}^{(i)} \in k^{\prime}\left[\left[x_{d}\right]\right] .
$$

and assume that

$$
x_{1}^{\alpha_{1}} \cdots x_{d-1}^{\alpha_{d-1}} x_{d}^{s}
$$

is a monomial with non-zero coefficient in this expression (i.e. assume that $\left.\left(\alpha_{1}, \ldots, \alpha_{d-1}, s\right) \in \operatorname{Supp}\left(f_{N_{i}}\right)\right)$. The claim in 1) is that $l\left(\alpha_{1}, \ldots, \alpha_{d-1}, s\right) \geq$ $a N_{i}$. In fact, if $l\left(\alpha_{1}, \ldots, \alpha_{d-1}, s\right)<a N_{i}$, then $|\alpha|:=\alpha_{1}+\cdots+\alpha_{d-1}<N_{i}$ and $\frac{s}{N_{i}-|\alpha|}<\lambda(6.12 .4)$. But in such case

$$
s l_{V^{\prime}}\left(f_{N_{i}}\right) \leq \frac{s}{N_{i}-|\alpha|}<\lambda=\lambda_{\text {Coeff }\left(\mathcal{G}_{1}\right)}=\min \left\{s l_{V^{\prime}}\left(f_{N_{j}}\right) \mid 1 \leq j \leq s\right\},
$$

which is a contradiction. 
Both conditions 2) and 3) follow similarly, from (6.12.3) and ((6.12.4).

Set $S=k^{\prime \prime}[[t]]$ for some field extension $k^{\prime \prime}$ of $k^{\prime}$, and define

$$
\beta: k^{\prime}\left[\left[x_{1}, \ldots, x_{d}\right]\right] \rightarrow k^{\prime \prime}[[t]]
$$

the continuous morphism, such that $\beta\left(x_{i}\right)=\lambda_{i} t^{a}\left(\lambda_{i} \in k^{\prime \prime}\right)$, for $1 \leq i \leq d-1$, and $\beta\left(x_{d}\right)=t^{b}$.

So $\beta\left(\mathcal{G}_{1}\right)$ is the Rees algebra in $k^{\prime \prime}[[t]][W]$ generated by $\left\{\beta\left(f_{N_{i}}\right) \cdot W^{N_{i}} \mid 1 \leq\right.$ $i \leq s\}$.

It follows that for $k^{\prime \prime}$ an infinite field, and for sufficiently general $\lambda_{i} \in k^{\prime \prime}$ :

1') $\beta\left(f_{N_{i}}\right)$ has order at least $a N_{i}$ in $k^{\prime \prime}[[t]]$.

2') $\beta\left(f_{N_{i_{0}}}\right)$ has order $a N_{i_{0}}$ for some $f_{N_{i_{0}}} \cdot W^{N_{i_{0}}} \in \mathcal{F}_{1}$.

3') $\beta\left(g_{M_{j_{0}}}\right)$ has order strictly smaller then $a M_{j_{0}}$.

Finally Claim 1 in Remark 6.8, where now $\lambda_{\beta\left(\mathcal{G}_{1}\right)}=a$, asserts that $\beta\left(g_{M_{j_{0}}}\right) \cdot W^{M_{j_{0}}}$ is not integral over $\beta\left(\mathcal{G}_{1}\right)$; so (6.12.3) can not hold.

The following Theorem can also be proved using Hironaka's theory on infinitely near points in [7]; a theory based on the behavior by monoidal transforms. Our proof relies on the previous development in this section, which will also be used for the proof of Theorem 6.14.

Theorem 6.13. Let $\mathcal{G}_{1} \subset \mathcal{G}_{2}$ be an inclusion of Rees algebras on a smooth scheme $V$. Let $G\left(\mathcal{G}_{i}\right)$ be the Diff-algebra spanned by $\mathcal{G}_{i}(i=1,2)$. If $\mathcal{G}_{1} \subset \mathcal{G}_{2}$ is a finite extension, then $G\left(\mathcal{G}_{1}\right) \subset G\left(\mathcal{G}_{2}\right)$ is a finite extension.

Proof. The inclusion $G\left(\mathcal{G}_{1}\right) \subset G\left(\mathcal{G}_{2}\right)$ is clear. We will argue locally at a point $x \in \operatorname{Sing}\left(\mathcal{G}_{1}\right)$, and we make use of the criterion in Proposition 6.11 to show that the extension is finite. Let $\mathcal{F}_{1}=\left\{f_{N_{1}} \cdot W^{N_{1}}, \ldots, f_{N_{s}} \cdot W^{N_{s}}\right\}$ generate $\mathcal{G}_{1}$ locally at $x$, and let $\mathcal{F}_{2}=\left\{g_{M_{1}} \cdot W^{M_{1}}, \ldots, g_{M_{t}} \cdot W^{M_{t}}\right\}$ generate $\mathcal{G}_{2}$.

Set $V^{\prime} \stackrel{\pi}{\longrightarrow} V$ where $V^{\prime}$ is one dimensional, and let $x^{\prime} \in V^{\prime}$ map to $x$. Locally at $x^{\prime}$ one can factor $\pi$ as $V^{\prime} \subset V^{\prime \prime} \stackrel{\phi}{\longrightarrow} V$, so that $\phi: V^{\prime \prime} \rightarrow V$ is smooth. Let $\phi^{-1}\left(\mathcal{G}_{1}\right), \phi^{-1}\left(\mathcal{G}_{2}\right)$ denote the total transforms of $\mathcal{G}_{1}, \mathcal{G}_{2}$; and $\phi^{-1}\left(G\left(\mathcal{G}_{1}\right)\right), \phi^{-1}\left(G\left(\mathcal{G}_{2}\right)\right)$ be the total transforms of $G\left(\mathcal{G}_{1}\right), G\left(\mathcal{G}_{2}\right)$.

If $\left\{x_{1}, \ldots, x_{d}\right\}$ is a regular system of parameters at $\mathcal{O}_{V, x}$, then $\left\{x_{1}, \ldots, x_{d}\right\}$ extends to a regular system of parameters, say $\left\{x_{1}, \ldots, x_{d}, \cdots, x_{e}\right\}$ at $\mathcal{O}_{V^{\prime \prime}, x^{\prime}}$. It is easy to check that

(1) $\mathcal{F}_{1}=\left\{f_{N_{1}} \cdot W^{N_{1}}, \ldots, f_{N_{s}} \cdot W^{N_{s}}\right\}$ generate $\phi^{-1}\left(\mathcal{G}_{1}\right)$ locally at $\mathcal{O}_{V^{\prime \prime}, x^{\prime}}$;

(2) $\mathcal{F}_{2}=\left\{g_{M_{1}} \cdot W^{M_{1}}, \ldots, g_{M_{t}} \cdot W^{M_{t}}\right\}$ generate $\phi^{-1}\left(\mathcal{G}_{2}\right)$ at $\mathcal{O}_{V^{\prime \prime}, x^{\prime}}$.

(3) $\phi^{-1}\left(G\left(\mathcal{G}_{1}\right)\right)$ is the Diff-algebra generated by $\phi^{-1}\left(\mathcal{G}_{1}\right)$.

(4) $\phi^{-1}\left(G\left(\mathcal{G}_{2}\right)\right)$ is the Diff-algebra generated by $\phi^{-1}\left(\mathcal{G}_{2}\right)$. 
Therefore the setting at $V$ and at $V^{\prime \prime}$ is the same, and hence, in order to apply Proposition 6.11 we need only to show that given a finite extension $\mathcal{G}_{1} \subset \mathcal{G}_{2}$, the restrictions of the Diff-algebras $G\left(\mathcal{G}_{i}\right), i=1,2$, to a smooth one dimensional scheme $V^{\prime}$, have the same integral closure.

Lemma 6.6 says that the restriction of $G\left(\mathcal{G}_{i}\right)$ to $V^{\prime}$ is the Diff-algebra generated by $\operatorname{Coeff}\left(\mathcal{G}_{i}\right)(i=1,2)$. Remark 6.8 shows that for each index $i=1,2$, the Rees algebra $\operatorname{Coeff}\left(\mathcal{G}_{i}\right)$, and the Diff-algebra generated by Coeff $\left(\mathcal{G}_{i}\right)$, have the same integral closure. So it suffices to show that Coeff $\left(\mathcal{G}_{1}\right)$ and Coeff $\left(\mathcal{G}_{2}\right)$ have the same integral closure, which was proved in Proposition 6.12. In fact, (1), (2), (3) and (4) show that the setting of Proposition 6.12 holds.

Theorem 6.14. Let $\mathcal{G}_{1} \subset \mathcal{G}_{2}$ be an inclusion of Rees algebras on a smooth scheme $V$. Fix a smooth sub-scheme $Z \subset V$, and a local (or formal) retraction $V \rightarrow Z$. If $\mathcal{G}_{1} \subset \mathcal{G}_{2}$ is a finite extension, then $\operatorname{Coeff}\left(\mathcal{G}_{1}\right) \subset \operatorname{Coeff}\left(\mathcal{G}_{2}\right)$ is also finite.

Proof. Set $\pi: C \rightarrow Z$ where $C$ is smooth and one dimensional, and let $x^{\prime} \in C$ map to $x$. Locally at $x^{\prime}$, one can factor $\pi$ as $C \subset Z_{1} \stackrel{\phi}{\longrightarrow} Z$, so that $\phi: Z_{1} \rightarrow Z$ is smooth. The retraction of $V$ on $Z$, together with the morphism $Z_{1} \rightarrow Z$, define by fiber products, a retraction say $V_{1} \rightarrow Z_{1}$, and a smooth morphism, say $V_{1} \rightarrow V$.

The total transform of $\mathcal{G}_{1} \subset \mathcal{G}_{2}\left(\subset \mathcal{O}_{V}[W]\right)$ to say $\mathcal{G}_{1}^{\prime} \subset \mathcal{G}_{2}^{\prime} \subset \mathcal{O}_{V_{1}}[W]$ is again finite, and the construction of Coeff $\subset \mathcal{O}_{Z}[W]$ is compatible with base change. So

$$
\operatorname{Coeff}\left(\mathcal{G}_{1}^{\prime}\right) \subset \operatorname{Coeff}\left(\mathcal{G}_{2}^{\prime}\right) \subset \mathcal{O}_{Z_{1}}[W]
$$

is the total transform of

$$
\operatorname{Coeff}\left(\mathcal{G}_{1}\right) \subset \operatorname{Coeff}\left(\mathcal{G}_{2}\right) \subset \mathcal{O}_{Z}[W]
$$

By further restriction of $Z_{1}$ to $C$, we may assume that $Z_{1}$ is one dimensional. Theorem 6.14 follows now from Proposition 6.12 .

Theorem 6.15. Let $\mathcal{G}(=G(\mathcal{G}))$ be a differential algebra defined on a smooth scheme $V$. Fix a point $x \in \operatorname{Sing}(\mathcal{G})$, a smooth sub-scheme $Z \subset V$ containing $x$, and two local (or formal) retractions, say $\pi: V \rightarrow Z$ and $\pi^{\prime}: V \rightarrow Z$ at $x$. If $\operatorname{Coeff}(\mathcal{G})$ and $\operatorname{Coeff}(\mathcal{G})^{\prime}$ are defined in terms of $\pi$ and $\pi^{\prime}$ respectively, then both define same differential algebra in $\mathcal{O}_{Z, x}[W]$.

Proof. Let $G(\mathcal{G})$ denote the differential algebra spanned by $\mathcal{G}$ in the smooth scheme $V$. The claim is a corollary of 6.6 . 


\section{Further applications}

There is a particular but natural morphism among smooth schemes, namely that defined by blowing up closed and smooth centers (i.e. monoidal transformations). Given an ideal in a smooth scheme, there are several notions of transformations of sheaves of ideals defined in terms of monoidal transformations (e.g. total transforms, weak transforms, and strict transforms of ideals.).

Questions as resolution of singularities, or Log principalization of ideals, are formulated in terms of these notions of transformations. In the case of schemes over fields of characteristic zero, both resolution and Log principalization of ideals are two well known theorems due to Hironaka. If two ideals have the same integral closure, then a Log-principalization of one of them is also a Log-principalization of the other; the key point being that the transforms of both ideals also have the same integral closure.

Notions of transformations on ideals extend naturally to Rees algebras. And again, if two Rees algebras have the same integral closure, then their transforms are Rees algebras with the same integral closure.

Both theorems of Log-principalization of ideals and resolution of singularities are proved by induction on the dimension of the ambient space. In the setting of differential algebras this form of induction relates to the notion of restriction on a smooth sub-schemes, say $Z \subset V$ in Theorems 6.14.

The outcome of Theorems 6.15 is that such form of restriction on $Z$ is, up to integral closure, independent of the particular retraction. This result has been applied in [1], and plays a role in the extension of resolution theorems to Rees algebras. We refer here to [7] or [8] for the notions of transformations of differentiable algebras, and related results.

The results in this paper were also applied in [19], in relation with the study of hypersurface singularities over fields of positive characteristic.

\section{References}

[1] Encinas, S. and Villamayor, O.: Rees algebras and resolution of singularities. In Actas del "XVI Coloquio Latinoamericano de Álgebra" (Colonia del Sacramento, Uruguay, 2005), 1-24. Biblioteca de Rev. Mat. Iberoamericana. Rev. Mat. Iberoamericana, Madrid, 2007.

[2] Fernández-Lebrón, M. and Narváez-Macarro, L.: Hesse-Schmidt derivations and coefficient fields in positive characteristics. J. Algebra 265 (2003), no. 1, 200-210.

[3] Giraud, J.: Sur la théorie du contact maximal. Math. Z. 137 (1974), 285-310. 
[4] Giraud, J.: Contact maximal en caractéristique positive. Ann. Scien. École Norm. Sup. (4) 8 (1975), 201-234.

[5] Grothendieck, A. And Dieudonné, J.: Éléments de géométrie algébrique. IV. Étude locale des schémas et des morphismes de schémas IV. Inst. Hautes Études Sci. Publ. Math. 32. Press Univ. de France, Paris, 1967.

[6] Hironaka, H.: Idealistic exponents of a singularity. In Algebraic geometry (J. J. Sylvester Sympos., Johns Hopkins Univ., Baltimore, 1976), 52-125. John Hopkins University Press, Baltimore, Md., 1977.

[7] Hironaka, H.: Theory of infinitely near singular points. J. Korean Math. Soc. 40 (2003), no. 5, 901-920.

[8] Hironaka, H.: Three key theorems on infinitely near singularities. In Singularités Franco-Japonaises, 87-126. Sémin. Congr. 10. Soc. Math. France, Paris, 2005.

[9] Kawanoue, H.: Toward resolution of singularities over a field of positive characteristic. I. Foundation; the language of the idealistic filtration. Publ. Res. Inst. Math. Sci. 43 (2007), no. 3, 819-909.

[10] KolláR, J.: Lectures on resolution of singularities. Annals of Mathematics Studies 166. Princeton University Press. Princeton, NJ, 2007.

[11] Lejeune Jalabert, M. And Teissier, B.: Cloture intégrale des idéaux et équisingularité. Michigan Math. J. 28 (1981), 97-116.

[12] Lipman, J. And Teissier, B.: Pseudorational local rings and a theorem of Briançon-Skoda about integral closures of ideals. Michigan Math. J. 28 (1981), 97-116.

[13] Matsumura, H.: Commutative Algebra. Second edition. Mathematics Lecture Notes Series. Benjamin, Cumming Publishing Company, 1980.

[14] Mount, K. R. and Villamayor SR., O.: Taylor Series and higher derivations. Impresiones del Departamento de Matematicas 18. Universidad de Buenos Aires, 1968 (reimpresión 1979).

[15] NarváEz-Macarro, L: A note on the behaviour under a ground field extension of quasicoefficient fields. J. London Math. Soc. (2) 43 (1991), $12-22$.

[16] Traves, W.: Localization of the Hasse-Schmidt algebra. Canad. Math. Bull. 46 (2003), no. 2, 304-309.

[17] Vasconcelos, W. V.: Arithmetic of blowup algebras. London Mathematical Society. Lecture Note Series 195. Cambridge University Press, Cambridge, 1994.

[18] Villamayor, O.: Differential operators on smooth schemes and embbeded singularities. Rev. Un. Mat. Argentina 46 (2005), no. 2, 1-18.

[19] Villamayor, O.: Hypersurface singularities in positive characteristic. Adv. in Math. 213 (2007), no. 2, 687-733. 
[20] WŁodarczyk, J.: Simple Hironaka resolution in characteristic zero. J. Amer. Math. Soc. 18 (2005), 779-822.

[21] Yakutieli, A.: An explicit construction of the Grothendieck residual complex. Astérisque 208. Soc. Math. France, 1992.

[22] Youssin, B.: Newton Polyhedra without coordinates. Mem. Amer. Math. Soc. 87 (1990), no. 433, i-vi, 1-74.

[23] Zariski, O. and Samuel, P.: Commutative Algebra. Vol II. The University Series in Higher Mathematics. D. Van Nostrand Co., Princeton, N. J.-Toronto-London-New York, 1960.

Recibido: 4 de julio de 2006

Orlando Villamayor U. Departamento de Matemáticas Facultad de Ciencias Universidad Autónoma de Madrid Cantoblanco, 28049 Madrid, Spain villamayor@uam.es 